CHAPTER 11

\title{
G Protein-Coupled Receptors: Conformational "Gatekeepers" of Transmembrane Signal Transduction and Diversification
}

\author{
RAVINDER ABROL* AND WILLIAM A. \\ GODDARD III, FRSC*
}

Materials and Process Simulation Center (MC 139-74), California Institute of Technology, 1200 E California Blvd, Pasadena, CA 91125, USA

\subsection{Introduction}

Cells are the fundamental units of all known life forms. This central role requires the cells to sense and interact with their environment or other cells and produce a physiological response vital to the proper functioning of the cell. Cellular signaling machinery is comprised of different proteins that enable and drive the amazing plethora of functions necessary for growth, survival and eventual death at the cellular level and in turn at the organism level (for multi-cellular life).

Cells need to process signals produced both inside and outside the cell. The extracellular signals can originate from other cells in the same organism (e.g. adrenaline), other organisms (e.g. pheromones) or the environment (e.g. photons, tastants, etc.). Cellular signaling proteins have evolved to sense this diverse set of signals either via direct contact with the signals

\footnotetext{
RSC Drug Discovery Series No. 10

Extracellular and Intracellular Signaling

Edited by James D. Adams, Jr. and Keith K. Parker

(C) Royal Society of Chemistry 2011

Published by the Royal Society of Chemistry, www.rsc.org
} 
(e.g. binding to the signaling molecule) or via binding to other proteins that are in direct contact with the signals. Different cells are also programmed to sense different signals depending on the proteins expressed in their plasma membrane. These membrane proteins not only enable signal transmission, but also signal modulation and diversification by undergoing conformational changes and/or through the specificity of protein-protein interactions.

Transmembrane (TM) signal transduction by membrane proteins is the dominant component of cellular signaling, as it enables a cell to convert an extracellular signal into one or more intracellular signals or responses. There are three main classes of membrane proteins that enable TM signal transduction: ion-channel receptors, enzyme-linked receptors and $G$ protein-coupled receptors (GPCRs), with GPCRs playing the most prominent role.

In this chapter, we will focus on GPCRs and use current structural insights from experiments and computational predictions to describe the spectacular role played by conformationally malleable GPCRs in sensing extracellular signals, which cover a broad spectrum from photons (for vision), chemosensory ligands (e.g. for taste and smell) and neurotransmitters/hormones (e.g. dopamine, serotonin, adrenaline, acetylcholine) to peptides (e.g. cytokines) and larger proteins. Being receptors for neurotransmitters, hormones and cytokines, GPCRs have been implicated in a majority of disease processes, which has made them a very attractive target for therapeutic applications, as blocking or activating these receptors by antagonists or agonists respectively blocks or activates a whole cascade of signaling events. However, different subtypes of a GPCR (capable of sensing the same agonist) might induce a different or even opposite response to the signal depending on the cell type and the location in the organism. This is one of the many reasons that most drugs have side-effects and the lack of complete knowledge about the signaling pathways being manipulated by drugs makes it difficult to ascertain whether some of the side-effects are caused by off-target proteins (other proteins or other subtypes of the target protein) or by the target protein itself. Designing GPCR drugs with no or minimal side-effects is one of the biggest challenges in the rational design of GPCR targeting therapeutics.

This chapter contains a brief section on cellular signaling to provide some background and context for GPCR-mediated signal transduction followed by a detailed section on GPCRs, which is divided into subsections on their structure, signal-induced conformation driven signal transduction (amplification and diversification), biased signaling induced by extracellular ligands and challenges in rational design of GPCR targeting drugs. The focus of the chapter is the processing and modulation of extracellular signals by GPCRs, so discussion on intracellular signaling pathways will be limited and will only focus on their direct connection to GPCR activation. The chapter concludes with a brief section on the discoveries and challenges that lie ahead for GPCR-mediated signaling. 


\subsection{Cellular Signaling}

Life of an organism at the biochemical level can be thought of as a collection of biological events, some occurring sequentially in time and some in parallel. Each of the biological events can, in turn, be broken down into one or more signaling cascades that usually consist of multiple signaling processes separated in space and time. How a specific cell in an organism will behave depends critically on this spatio-temporal separation of signaling processes. Cellular signaling broadly refers to these highly evolved networks of signaling events and cascades that allow a cell to function.

Cellular signaling has been studied for more than 100 years now and our knowledge of its complexity at multiple levels has been greatly enhanced through advances in many different areas of biology. ${ }^{1-3}$ It still appears that we may have barely opened the "Pandora's box" as the current knowledge seems unable to explain the beautiful richness of the complexity of life observed on land and especially in the oceans. One of the many great examples of signaling complexity manifested in nature is the dynamic camouflage ability of cuttlefish, where highly coupled signaling cascades enable these mollusks to replicate not only the color of their environment but also its visual pattern and texture (depth) onto their skin to blend in with that environment. ${ }^{4,5}$

\subsubsection{Types of Signaling}

Any signaling network or cascade is a series of biochemical processes, where each process is initiated by the appearance of a signal which is followed by its sensing, processing and transmission as another signal or signals for the next downstream process in the signaling cascade. The signal may appear either inside or outside the cell for processing. Extracellular signals are usually sensed and processed by plasma membrane proteins. Intracellular signals are processed by soluble proteins or membrane proteins in the plasma membrane or those on the surface or cell organelles.

The spatio-temporal separation of signaling processes and cascades mentioned earlier allows one to classify signaling processes into the following types that depend on the spatial origin of the signal in an organism and its reach within the organism:

a) Endocrine signaling: In this long-range signaling, signal molecules such as hormones are released by a cell and travel long distances (via bloodstream in animals or vascular system in plants) to cause an effect in a different part of the organism. Processing of sensory signals like light, taste and smell can also be considered endocrine.

b) Paracrine signaling: This is a short-range version of endocrine signaling, where the signal produced by a cell is sensed locally, e.g. neurotransmitters that are processed by proximal neurons.

c) Juxtacrine signaling: In this signaling process, the signal is membrane bound on one cell and is sensed by a receptor on the adjacent cell, 
e.g. membrane proteins on a cell membrane can be sensed by a Notch protein on the neighboring cell.

d) Autocrine signaling: In this signaling process, cells release a signal molecule outside the cell, which is sensed by a membrane protein on the same cell leading to self-stimulation, e.g. breast cancer cells release transforming growth factor alpha (TGF- $\alpha$ ) that interact with its epidermal growth factor (EGF) receptor.

e) Intracrine signaling: In this signaling process, the signal molecule is generated inside the cell and sensed by another receptor from inside the cell.

f) Electrical signaling: This specialized signaling process propagates an electrical potential along the length of the cell and occurs on a long spatial scale. The cells that use this process are the neurons of the animal nervous system, which are unusually long cells.

Any signaling cascade may be made up of one or more of the abovementioned signaling processes.

\subsubsection{Membrane Proteins in Signaling}

The diversity of signals is immense. Chemical signals are molecules ranging greatly in size from the very small (like oxygen molecule, adrenaline, etc.), to peptides (like cytokines) and large proteins. Non-chemical signals include photons that are absorbed by cis-retinal-rhodopsin complex in the retina and initiate a cascade of processes that start in the cell and end in the brain with the perception of vision. Different proteins have evolved along with the signaling processes to sense this broad spectrum of signals.

Spatial separation of signaling cascades in an organism is achieved by cells expressing different receptors on their surface as well as inside the cell. Cell surface receptors (membrane proteins) enable signal transduction across the plasma membrane by converting an extracellular signal into one or more intracellular signaling cascades. Three main classes of membrane proteins dominate TM signal transduction: ${ }^{6}$

a) Ion-channel receptors (ICRs): These proteins are responsible for sensing neurotransmitter molecules or voltage gradients across the membrane, as upon binding to the signal molecules or sensing the membrane potential these receptors undergo a conformational change that opens or closes a channel and allows specific ions to cross the plasma membrane. ${ }^{7}$

b) Enzyme-linked receptors (ELRs): These are a diverse class of single-pass TM proteins that contain an extracellular ligand binding site and an intracellular catalytic/enzyme-binding site with a guanylyl cyclase, phosphatase, serine/threonine kinase or tyrosine kinase activity. Receptor tyrosine kinases dominate this class. ${ }^{8}$

c) G protein-coupled receptors (GPCRs): These form the largest superfamily of membrane proteins that undergo "signal-specific" conformational changes upon activation by a diverse set of extracellular signals. These 
conformational changes in the receptor are transmitted to cytoplasmic $\mathrm{G}$ proteins and $\beta$-arrestins for downstream signal transmission and potential diversification for a physiological response as will be discussed later.

GPCRs are integral membrane proteins with an extracellular N-terminus and seven TM helices connected by loop regions. They use their N-terminus, extracellular loops and extracellular facing TM portions to sense their signals. As a single protein family, they interact with the most diverse set of signals from sensory signals (vision, taste, smell, pheromones, etc.) to large signal molecules (other proteins).

GPCRs are the focus of this chapter because being embedded in the plasma membrane they sit at the top of complex signaling cascades as gatekeepers and use their conformational flexibility to amplify, diversify and select downstream signaling pathways inside the cell with amazing specificity. This role has also implicated them in almost all disease mechanisms ${ }^{9}$ and about $30 \%$ of the approved drugs use them as targets to block or activate a whole signaling pathways in cells. ${ }^{10}$

In the next section we will describe what is known about GPCRs, both experimental and computational efforts to determine their structures, biochemical studies probing their downstream signaling effects, structural as well functional implications of their signal sensing (ligand binding) properties and challenges in drug design aimed at GPCR targets.

\subsection{G Protein-Coupled Receptors}

GPCRs are integral membrane proteins with seven TM helices connected by three extracellular loops (ECLs) and three intracellular loops (ICLs). They form the largest superfamily in the human genome with 800 GPCRs identified, including $\sim 370$ non-sensory receptors. ${ }^{11}$ A variety of bioactive molecules, including biogenic amines, peptides, lipids, nucleotides, hormones and proteins modulate GPCR activity to effect regulation of essential physiological processes (e.g. neurotransmission, cellular metabolism, secretion, cell growth, immune defense and differentiation). Thus, many important cell recognition and communication processes involve GPCRs. Due to mediating numerous critical physiological functions, GPCRs are involved in all major disease areas including cardiovascular, metabolic, neurodegenerative, psychiatric, cancer and infectious diseases. ${ }^{9}$ GPCRs represent $30-50 \%$ of the current drug targets for activation (by agonist drugs) or inhibition (by antagonists or inverse agonists). It is estimated that the $\sim 80$ GPCR-targeting drugs currently marketed account for $\sim \$ 50$ billion annual sales. Many of these drugs have annual sales $>\$ 2$ billion. Target evaluation, lead identification and optimization of GPCR assays have accelerated progress in identifying multiple subtypes for many GPCRs with specific cell and tissue functions. A detailed structural understanding of their function (activation) will have a tremendous and broad impact in many areas. ${ }^{12}$ 


\subsubsection{Structure of GPCRs}

GPCRs can be organized into six families (acronymed GRAFTS, a minor variation on the original GRAFS classification proposed ${ }^{13}$ based on the phylogenetic criteria): glutamate, rhodopsin, adhesion, frizzled, taste 2 and secretin.

a) Rhodopsin family (also called Class A or Family 1): This diverse family dominates the human GPCRs with $\sim 670$ members (out of $\sim 800$ total). The family is further divided into four subfamilies $-\alpha, \beta, \gamma, \delta$. The $\alpha$ subfamily includes light-sensing rhodopsin receptor, biogenic amine (dopamine, serotonin, histamine, muscarinic) receptors as well as cannabinoid and prostanoid receptors among others. The $\beta$ subfamily mainly consists of peptide-binding proteins. The $\gamma$ subfamily receptors bind to peptides or lipid-like molecules, some examples being chemokine, angiotensin, somatostatin and opiod receptors. The $\delta$ subfamily is dominated by olfactory receptors $(\sim 388$ out of $\sim 670$ total in rhodopsin family) and also contains purinergic and glycoprotein-binding receptors. The vomeronasal pheromone receptors putatively also belong to the rhodopsin family. Being the largest family, it is not surprising that this family is targeted by the majority of GPCR drugs.

b) Secretin/Adhesion family (also called Class B or Family 2): The secretin receptors of this family bind peptide hormones, whereas Adhesion receptors bind to extracellular matrix molecules based on the knowledge of receptors de-orphaned so far. ${ }^{13}$

c) Glutamate family (Class C or Family 3): This family consists of metabotropic glutamate receptors, $\gamma$-aminobutyric acid $\mathrm{B}\left(\mathrm{GABA}_{\mathrm{B}}\right)$ receptors, sweet and umami (due to glutamate in monosodium glutamate or MSG, a food additive) taste receptors and calcium-sensing receptor. One of the two taste receptor monomers (T1R1, T1R2) combines with a third monomer (T1R3) to form functional heterodimers for sweet taste (T1R2 + T1R3) or umami taste (T1R1 + T1R3). ${ }^{14}$

d) Frizzled family: This family consists of $\sim 10$ frizzled receptors (which bind to Wnt glycoproteins) and a smoothened receptor (which appears to function without binding to any ligand).

e) Taste 2 family: This family exclusively consists of $\sim 25$ bitter taste receptors, ${ }^{15-16}$ which share the sensing of different bitter tastants with a different subset of receptors. These taste receptors have recently been found in the gastrointestinal (GI) tract as well. ${ }^{17}$ Their function in the gut is not known but their activation (in mice) has been shown to activate gut hormonal receptors (cholecystokinin or CCK and peptide YY or PYY), ${ }^{18}$ which are coupled to the glucagon-like peptide 1 (GLP-1) and other glucose metabolism pathways.

The structural topology of the receptors within each of the families mentioned above appears to be similar based on structural and sequence analysis as shown in Box 11.1 (for Family 1, 2 and 3). GPCRs in general are not 


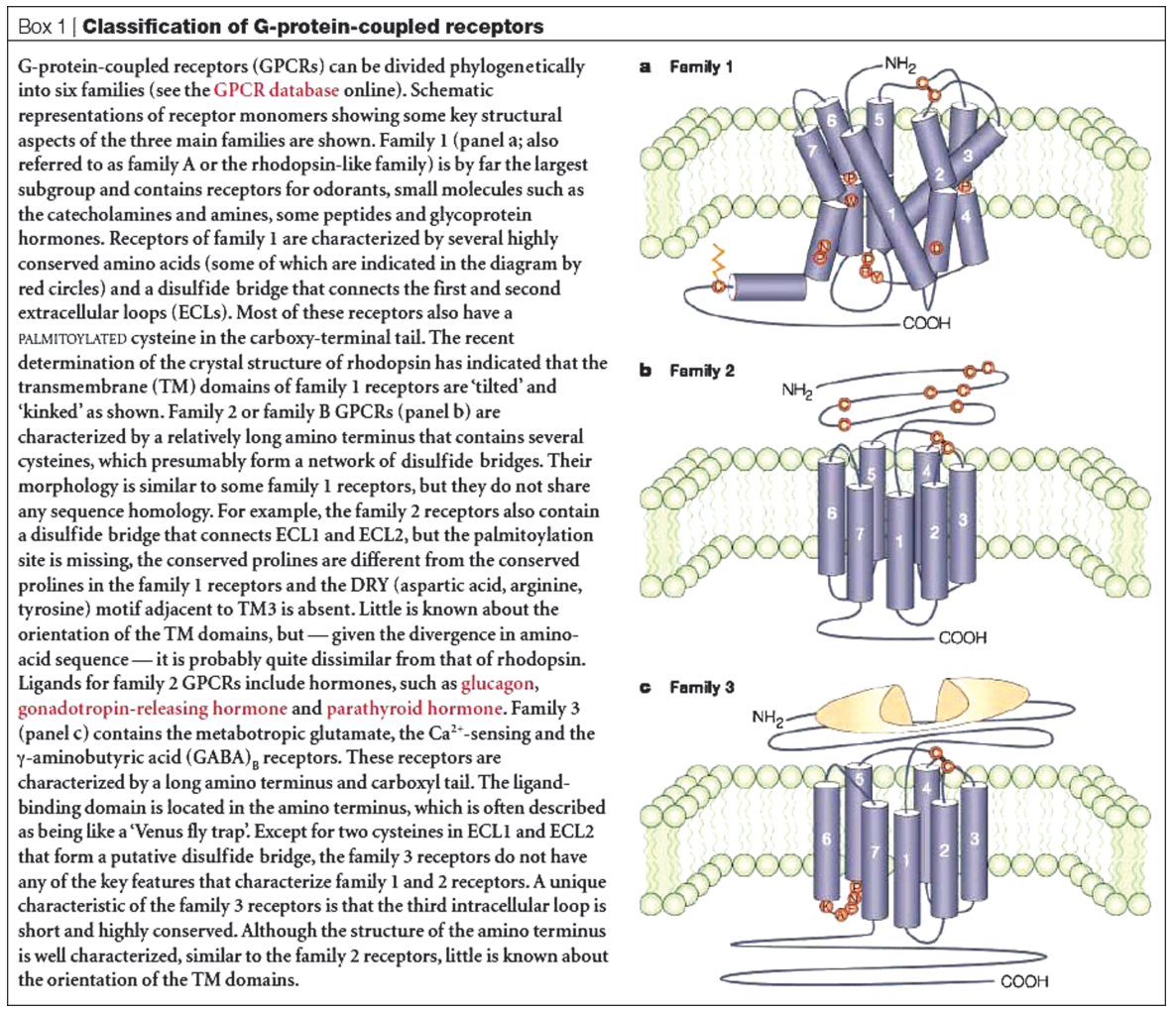

Box 11.1 Reprinted by permission from Macmillan Publishing Ltd.: George et al., Nat. Rev. Drug Discov., 1(10), 808-820. Copyright 2002.

homologous to each other unless they bind to the same ligands and, apart from the seven-TM helix topology, nothing appears to be common across all receptors.

Rhodopsin (Family 1) receptors share some common sequence motifs like D(E)RY at the bottom of TM3, WXPFF motif in TM6, NPXXY motif in TM7 and some conserved prolines usually in the middle of many TMs that produce kinks in their helices. Small molecule ligands typically bind in the extracellular facing half of the TM regions and peptides/proteins bind mainly to the extracellular loops and $\mathrm{N}$-terminus. There is a highly conserved disulfide bridge between cysteines in ECL2 and top of TM3.

Secretin/Adhesion (Family 2) receptors have a long N-terminal ectodomain that binds to ligands and contains many conserved cysteines, which can help the long N-terminus to form a stable tertiary structure (see Box 11.1). These receptors don't share any sequence motifs with Family 1 receptors even in the TM regions, so it is not obvious if they will have the same TM bundle topology of Family 1 receptors.

Glutamate (Family 3) receptors have a long N-terminus and a long Cterminus as well. Most receptors use their long $\mathrm{N}$-terminus to bind to their 
endogenous ligands and the binding pocket is sometimes referred to as the venus fly trap (or VFT; see Box 11.1).

Next we will describe the structure determination efforts aimed at GPCRs and what we have learnt from the available structures generated by these efforts and functional studies of GPCRs.

\subsubsection{Structure Determination}

The experimental structure determination of GPCRs had been quite slow until recently relative to other membrane proteins (and obviously soluble proteins) despite intense efforts by many protein crystallography and NMR groups (currently, six GPCR structures present in the PDB out of more than 65,000 structures). Until 2007, crystal structure was available only for bovine rhodopsin. ${ }^{19,20}$ This lack of structures was due to various factors including poor protein expression levels, difficulties in large-scale receptor purification, the insolubility in media-lacking phospholipids and other difficulties in crystallization. Significant technological advances in GPCR crystallization techniques have been made in the last few years that include emergence of lipidic cubic phase crystallization $^{21}$ and its coupling to the protein fusion methodology ${ }^{22}$ that replaces a disordered region of protein structure with T4-lysozyme to increase the surface area potential for crystal contacts.

These advances have resulted in the availability of crystal structures ${ }^{23}$ of two human GPCRs: $\beta 2$ adrenergic receptor ( $\beta 2 \mathrm{AR}$ ) bound to a partial inverse agonist ${ }^{24}$ and adenosine $\mathrm{A}_{2 \mathrm{~A}}$ receptor $\left(\mathrm{A}_{2 \mathrm{~A}} \mathrm{R}\right)$ bound to an antagonist. ${ }^{25}$ Other advances include increasing GPCR thermal stability by systematic mutagenesis (which led to the structure of turkey $\beta 1$ adrenergic receptor $(\beta 1 \mathrm{AR}){ }^{26}$ and optimization of receptor purification (which led to the structure of activated ligand-free bovine opsin structure by itself $^{27}$ and in association with a carboxyl-terminal peptide fragment of its $G_{\alpha}$ subunit transducin). ${ }^{28}$ Figure 11.1

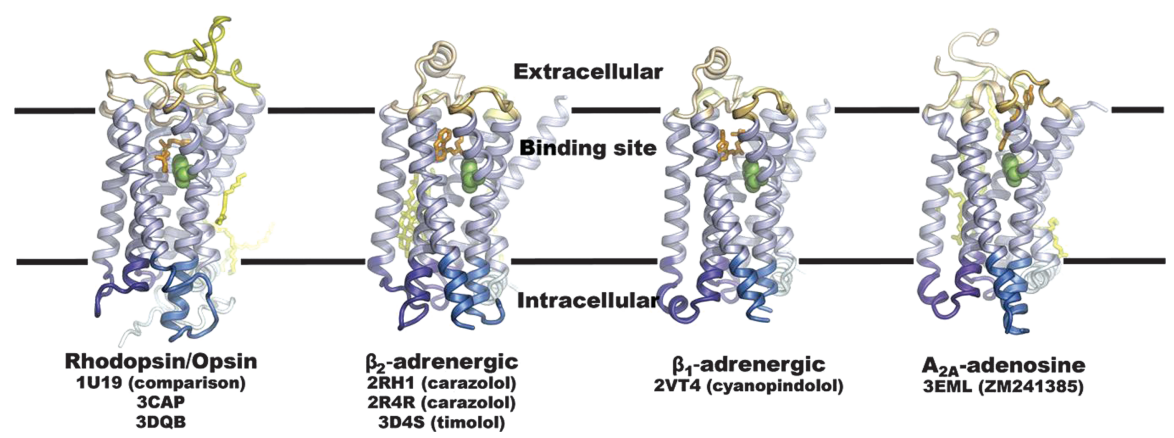

Figure 11.1 Four representative crystallized GPCRs solved to date. Reprinted from structure 17(1), Hanson and Stevens, Discovery of New GPCR Biology: One receptor Structure at a Time, 8-14, Copyright 2009 with permission from Elsevier. 
shows the structures of bovine rhodopsin, human $\beta 2$ adrenergic, turkey $\beta 1$ adrenergic and human adenosine $A_{2 A}$ receptors. ${ }^{23}$ They all share the same TM topology (relative positions of TM helices), but can differ (sometimes significantly) in helix tilts and rotations as will be discussed in the next section.

An invertebrate GPCR (squid rhodopsin) has also been crystallized, which showed unusually long TM regions 5 and $6 .{ }^{29}$ At least two more human GPCR structures are expected this year (dopamine D3 and chemokine CXCR4 receptors) and significantly more in the next decade. Progress is also being made in developing solid-state NMR techniques ${ }^{30}$ for GPCR structure determination. This rapid growth in GPCR crystal structures since 2007 is beginning to provide insight into the structural biology of these proteins, ${ }^{23,31}$ however, the progress is expected to remain slow due to intrinsic flexibility of these versatile receptors, which prevents them from packing into ordered crystals.

\subsubsection{Structural Diversity of Current GPCR Structures}

The seven-TM helix topology of GPCRs presents unique advantages and challenges for the quantification of sequence-structure relationships. Many comparative modeling programs can predict structures of globular proteins (with $30 \%$ or higher sequence identity to a crystallized protein) to a reasonable accuracy, as the belief is that a major fraction of structural folds is now known for globular proteins. The same cannot be said for membrane proteins in general. GPCRs, however, can be thought of as having one structural fold, consisting of seven TM helices interconnected by intracellular and extracellular loops. The TM helices display high sequence conservation as compared to the loop regions as expected (see Table 11.1).

The table shows the sequence identity (Table 11.1A) and sequence similarity (Table 11.1B) (similarity using BLOSUM62, ${ }^{32}$ where two residues are considered similar if the corresponding substitution element in the BLOSUM62 matrix is $>0$ ) for the five GPCR sequences that have been crystallized.

To quantify the relationship between sequence and structure for GPCRs, we need to characterize the known structures using some standard geometrical parameters. As crystal structures don't provide absolute membrane orientation of GPCRs, we use their orientation as predicted by the OPM (Orientation of Proteins in Membrane) database, ${ }^{33}$ which aligns each newly deposited membrane protein structure to an implicit membrane maximizing the free energy of membrane insertion. The middle of the membrane corresponds to the $z=0$ plane or the hydrophobic plane. Each GPCR structure can then be characterized by the six orientation parameters of the seven helices relative to this plane. Figure 11.2A shows how the helix position and tilt are defined. Helix position $(R)$ on the hydrophobic plane is then given by $x$ and $y$. Value $h$ corresponds to the hydrophobic center residue from the helix that will be 


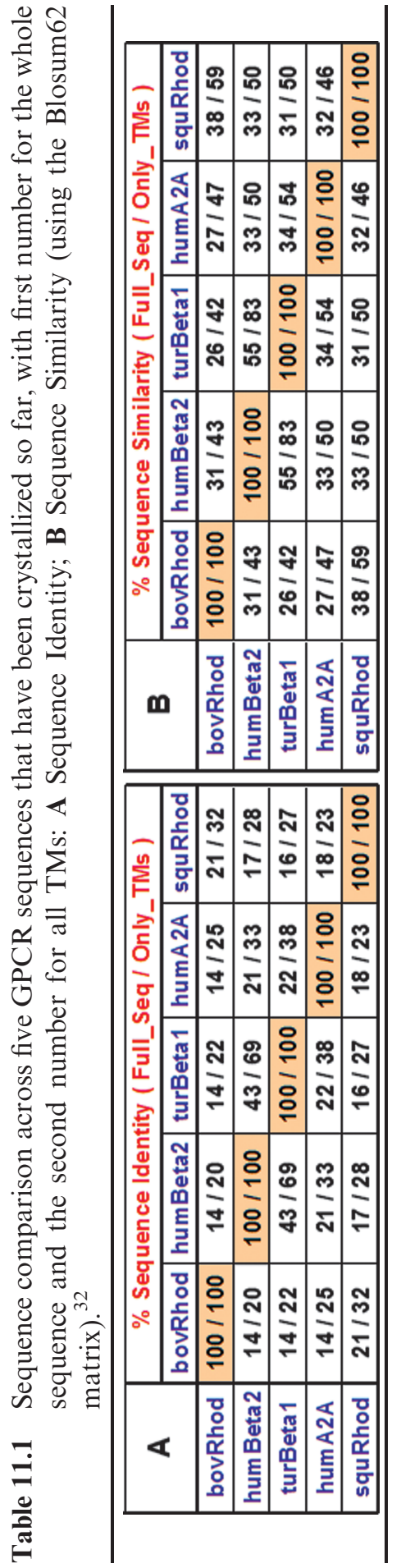




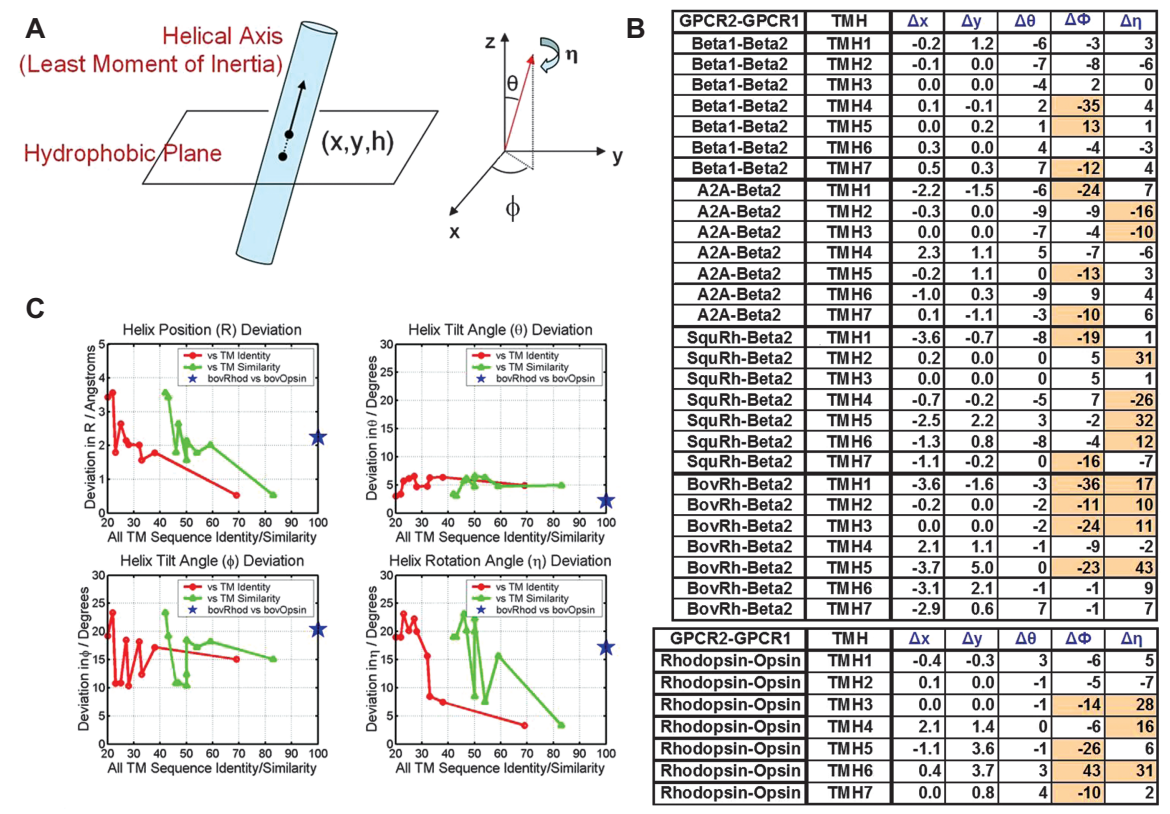

Figure 11.2 A Definition of the helical axis; B Table showing relative orientation parameters for the GPCRs with crystal structures; C Correlation of deviation in orientation parameters with sequence identity/similarity.

positioned on the hydrophobic plane. Two angles, $\theta$ and $\phi$, specify the tilt angles of the helix and the angle $\eta$ corresponds to the helix rotation angle about its axis. The two tilt angles $(\theta, \phi)$ and the rotation angle $(\eta)$ require a definition of the helical axis, which needs to account for the reality of bent helices as prolines are commonly found in the TM helices. We use a helical axis that corresponds to the lowest moment of inertia vector for the helix obtained by diagonalizing the moment of inertia matrix for the helix using only heavy backbone atoms.

We rotate the membrane-aligned GPCRs from the OPM database in the $x-y$ plane such that the helical axis of TM helix (TMH) 3 goes through the origin, and that of TMH 2 intersects the $x$-axis. Figure 11.2B shows the relative six orientation parameters for all seven helices for the crystallized GPCRs relative to $\beta 2$ adrenergic receptor and of bovine rhodopsin (cis-retinal bound form) relative to bovine opsin (the retinal free form) that is considered a conformation along the activation pathway of rhodopsin.

In order to correlate the sequence variability of these GPCRs with their helix geometries, we calculated all-to-all (across these systems) RMS (root-meansquared) deviations in position $R$ ( $x, y$ position in the $z=0$ plane), and angles $\theta, \phi, \eta$ averaged over all helices and plotted them against the corresponding sequence identity and similarity. The equations used for the deviations between 
a GPCR $i$ and a GPCR $j$ are:

$$
\begin{aligned}
R_{R M S D}^{i, j} & =\sqrt{\frac{1}{7} \sum_{k=1}^{7}\left[\left(x_{k}^{i}-x_{k}^{j}\right)^{2}+\left(y_{k}^{i}-y_{k}^{j}\right)^{2}\right]} \\
\alpha_{R M S D}^{i, j} & =\sqrt{\frac{1}{7} \sum_{k=1}^{7}\left(\alpha_{k}^{i}-\alpha_{k}^{j}\right)^{2}, \alpha=\theta, \phi, \text { or } \eta}
\end{aligned}
$$

The sum over index $k$ is for the seven TM helices. Figure 11.2C shows all-toall deviations (for all $i-j$ pairs of GPCRs) as a function of identity or similarity between GPCR $i$ and GPCR $j$, to highlight the variability seen in these five systems. In addition, the deviation of bovine rhodopsin and opsin is plotted (as a blue star symbol) to show the variability in functional conformations originating from a single GPCR sequence. At least across the GPCRs with known structure, we see that the deviations in helix position $(\mathbf{R})$ and helix rotations ( $\eta$ ) are inversely correlated to the closeness (identity or similarity) between sequences. The corresponding deviations in helix tilts $(\theta, \phi)$ appear to be independent of the sequence identity or similarity, with bigger deviations in the $\phi$ tilt angle. Absolute deviations of these tilt angles across the GPCRs can be inferred from Figure 11.2B and are roughly in the range $\pm 10^{\circ}$ for $\theta$ and $\pm 45^{\circ}$ for $\phi$. The deviation of bovine rhodopsin and opsin is at par with that of weakly related sequences as seen from the blue star symbol in Figure 11.2C. Overall, the deviations in helix orientation appear large except for receptors in the same family ( $\beta 1$ and $\beta 2$ ) and any structural prediction method should be able to sample these deviations in a complete way in order to identify all low-energy conformations, including the active conformations. Except for the differences between bovine rhodopsin and opsin, a clear structural view of the conformational changes that occur upon GPCR activation is still lacking. It is critical to understand these activation-related conformational changes because it will not only shed light on the function of GPCRs but will also provide a unique structural handle on designing better drugs through direct modulation of GPCR function.

\subsubsection{Prediction of GPCR Structure and Ligand Binding}

In the absence of experimental structure information for almost all GPCRs, protein structure prediction and modeling is playing an increasingly important role in providing detailed structural information that is relevant to their activation and ligand binding. Membrane proteins and their environment have been the focus of structure prediction and dynamics simulations for some years now. ${ }^{34}$ The interaction of these proteins with their lipid environment is considered critical to their in vivo folding and many recent studies have attempted to quantify this interaction on an absolute thermodynamic basis $^{35}$ by providing, for example, thermodynamic costs for the insertion of 
amino acids (that make up the TM helices) into the lipid bilayer. ${ }^{36}$ An implicit membrane potential of mean force has also been obtained recently for each amino acid as a function of the membrane normal using experimental structures of $\alpha$-helical membrane proteins. ${ }^{37}$ The 3D-structure of these $\alpha$ helical membrane proteins, to which GPCRs belong, is strongly affected by interhelical interactions (mainly $\mathrm{H}$-bonds and salt-bridges). ${ }^{38}$ An accurate structure-prediction methodology needs to be able to sample and describe these interhelical interactions very thoroughly.

Availability of a good structure (from experiment or modeling) allows for the molecular dynamics (MD) simulations to be performed on these proteins in their native lipid environment under ambient conditions. MD simulations of biomolecular systems have come of age and are contributing enormously to the understanding of their dynamical behavior. With more affordable and more powerful computers, the dynamics of these membrane proteins can be followed in their explicit lipid environment for hundreds of nanoseconds or more. This situation is only going to improve with time, allowing for dynamics over even longer timescales of the order of microseconds. However, the conformational changes that accompany GPCR activation are known to occur on the millisecond or higher timescales, suggesting that explicit all-atom dynamics will not be able to describe these large conformational changes for some years; coarse grained simulations may do it sooner.

Many methods have been used to obtain model structures for membrane proteins due to their pharmacological importance. These methods have been reviewed elsewhere. ${ }^{34,39}$ For GPCRs the main approach has been homology modeling (using the X-ray structure of bovine rhodopsin as a reference until 2007, and others more recently). Because of their low homology to other GPCRs of pharmacological interest, most studies have used constraints based on mutation and binding experiments coupled to the homologous rhodopsin structure to guide additional mutation experiments. These structures have not generally been sufficiently accurate for predicting binding sites of ligands. Methods are also available for predicting structures of membrane proteins in general. ${ }^{40}$

Our group has been developing de novo computational approaches (not based on homology) such as MembStruk and HierDock, ${ }^{41}$ for predicting the 3D structure of a GPCR, and its ligand binding sites. MembStruk method involved prediction of the TM regions, helix optimization based on TM regions, placement of optimized helices in a template (rhodopsin), followed by a local optimization of the helix rotations. These methods have been applied successfully to dopamine, ${ }^{42}$ adrenaline, ${ }^{43}$ muscarine, ${ }^{44}$ chemokine, ${ }^{45}$ prostaglandin $\mathrm{DP}^{46}$ and serotonin ${ }^{47}$ receptors. In all these cases, MembStruk was used to generate an ensemble of GPCR structures, out of which only one structure was carried forward for docking. After predicting the best structure for the GPCR, we used the HierDock procedure to locate the binding region and to predict the binding configuration in this region. Then we compared the predicted structures for the ligand-GPCR complex with experimental binding and mutation data and in some cases with experimental Structure-ActivityRelationship (SAR) data. 


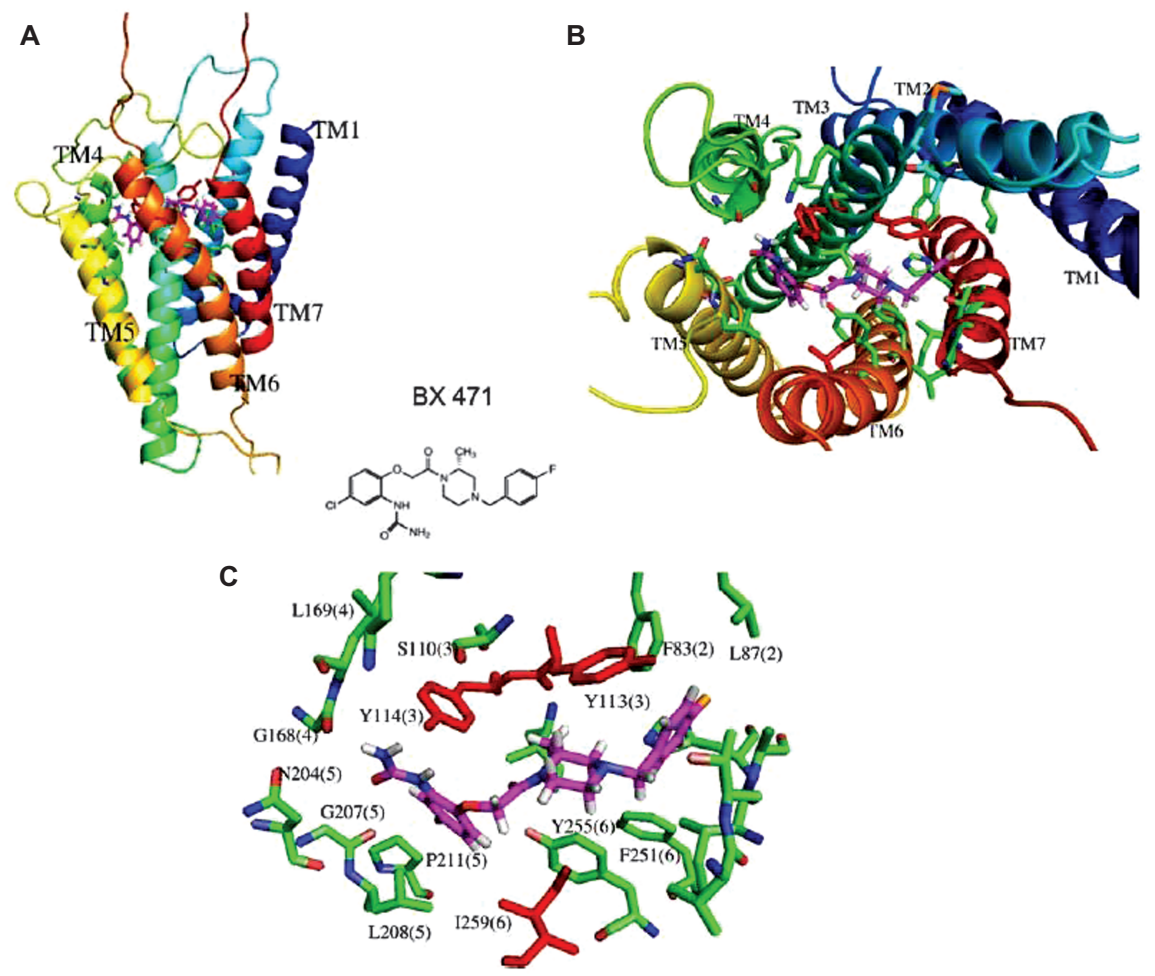

Figure 11.3 The predicted structure of human CCR1 bound to BX471 (from ref. 45). A Side view. B Top view. C Detailed binding site view.

As reviewed recently, ${ }^{48}$ this led to excellent results for modest-sized ligands such as dopamine and epinephrine and even for ligands such as haloperidol and domperidone. For example, Figure 11.3 shows the predicted structure for human CCR1 along with the binding site for its antagonist BX471. ${ }^{45}$ In this GPCR system, the mutagenesis studies were performed after the predictions, providing confidence in these prediction methods.

We have recently replaced the MembStruk method with the GEnSeMBLE (GPCR Ensemble of Structures in Membrane BiLayer Environment) method, which besides implementing an improved TM prediction step (PredicTM) contains a helix rotation optimization step (BiHelix) based on pairwise helix interactions that performs complete and thorough sampling of $\sim 1$ billion conformations in a highly efficient way (see Figure 11.4). This method was recently applied to predict the structure of human adenosine $\mathrm{A}_{2 \mathrm{~A}}$ receptor and its ligand binding site. ${ }^{49}$ The structural comparison of predicted and crystal structure is shown in Figure 11.5. The method was able to predict the ligand to within $2.8 \AA$ of the crystal pose and also identified 9 out of 12 protein residues in the binding site. 
a.

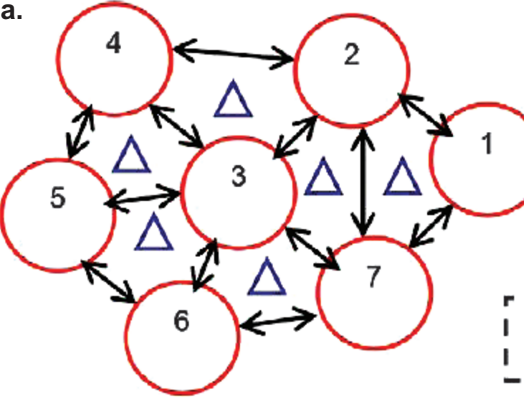

b.

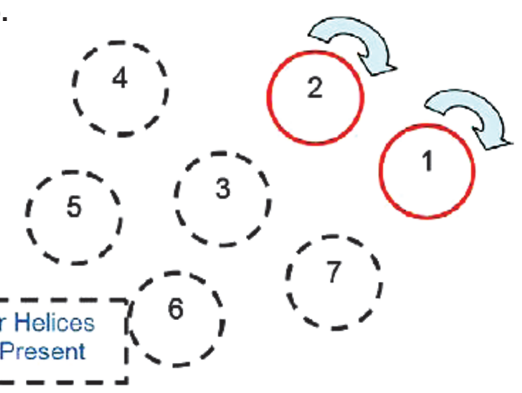

Figure 11.4 BiHelix sampling scheme, where sampling is done two helices at a time for all interacting helix pairs. a. All interacting helix pairs shown with a double arrow. b. Helix1-Helix2 optimization shown in the absence of other helices.

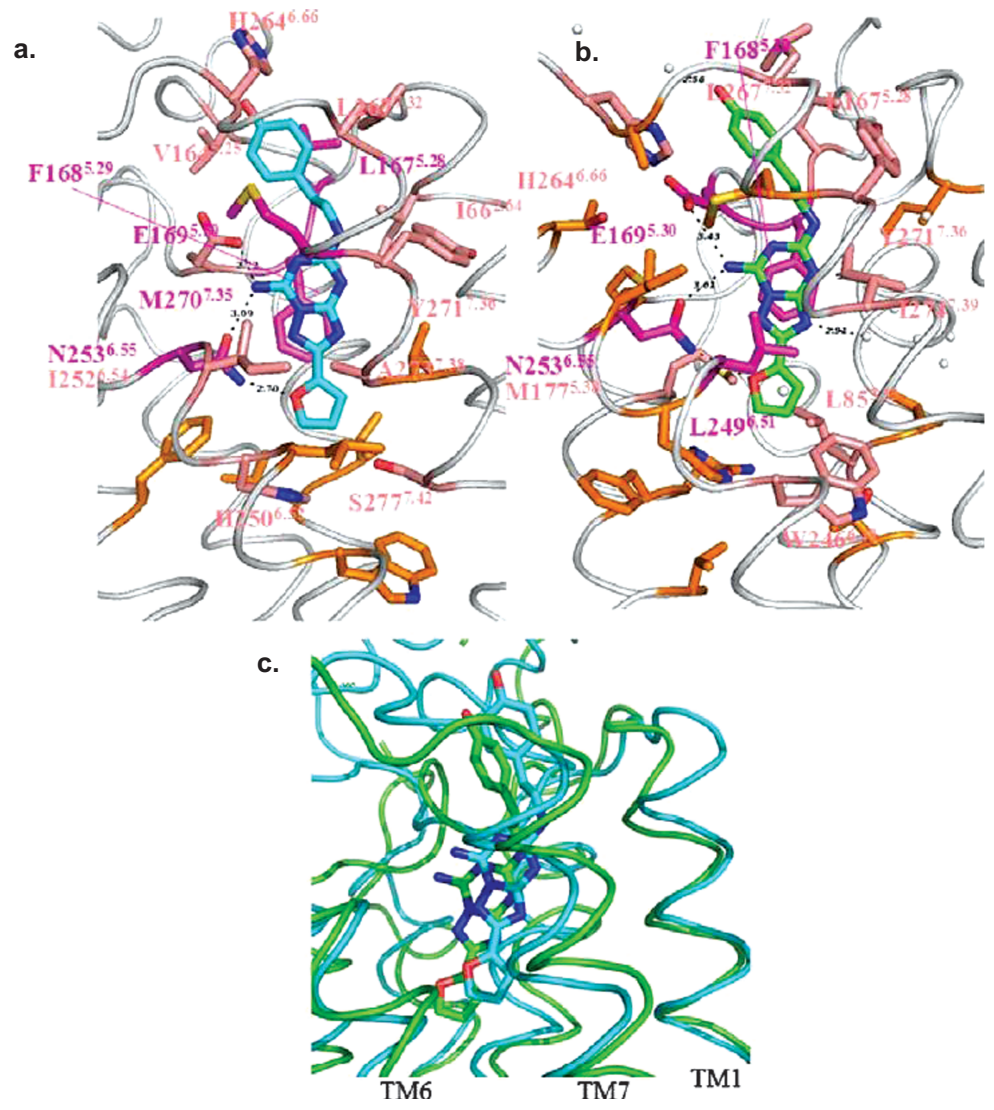

Figure 11.5 Predicted binding site of ZM241385 for human $\mathrm{A}_{2 \mathrm{~A}}$ adenosine receptor. a. Predicted pose. b. Crystal pose. c. Predicted and crystal poses overlaid. Reprinted with permission from Elsevier from Goddard et al., J. Struct. Biol., 170(1), 10-20. Copyright 2010. 


\subsubsection{GPCR Activation: Conformation Driven Functional Selectivity}

GPCRs undergo activation in response to an extracellular signal (e.g. an agonist ligand) and that information is relayed inside the cell through the coupling of the activated GPCR with cytoplasmic G proteins, which is normally followed by its coupling to $\beta$-arrestins that eventually ends in the internalization of the GPCR embedded into endosomes inside the cell. In that respect they can be called nature's allosteric robots that enable transduction of extracellular signals across the plasma membrane.

Proteins by their very nature are dynamic macromolecules and under physiological conditions exist in an ensemble of conformations. This dynamic motion can be considered molecular "breathing" "50 and is supported by NMR data as well as computer simulations. This motion can be visualized as that of a golf ball (protein) rolling on a golf course (the potential energy surface) being randomly kicked around (due to thermal fluctuations) by a force (the temperature, solvent, etc.). The bottom of the protein's multi-dimensional potential energy surface (corresponding to troughs on the golf course), called an energy well, can accommodate a micro-ensemble of isoenergetic and similar conformations. The greater the depth of an energy well, the more time the protein will spend in the conformations corresponding to that well. Favorable interaction with another molecule (e.g. a ligand or another protein) will change the character of the protein's potential energy surface and the energy wells resulting in a different ensemble of protein conformations that would prefer binding to that other molecule. Now we will see how these ideas can be applied to GPCRs.

\subsubsection{Multi-Conformational View of GPCRS}

One of the fundamental challenges in structure determination of GPCRs is their conformational flexibility. The Kobilka group has shown evidence for multiple conformational states in $\beta 2$ adrenergic receptor even in the presence of a single ligand, ${ }^{51}$ consistent with the energy landscape idea presented earlier. Debra Kendall's group has shown convincingly that a single mutation in the CB1 receptor can change the constitutively active receptor into the inactive form or the active form. ${ }^{52}$ This strongly suggests that even in the absence of any ligand, GPCRs are capable of major conformational changes with different functional outcomes.

These observations present a special problem in understanding structure and function of GPCRs: conformational changes in the protein structure are an essential aspect of its function. Thus, to understand GPCR activation we need to consider the multiple conformational states that the receptor can have under physiological conditions and we must consider the changes in these populations as the ligand interacts with them. This presents an enormous challenge to purely experimental structure determinations since the crystal must have all ligand-protein complexes identical, as evidenced by the fact that all currently available GPCR structures are in their inactive form (except opsin). 


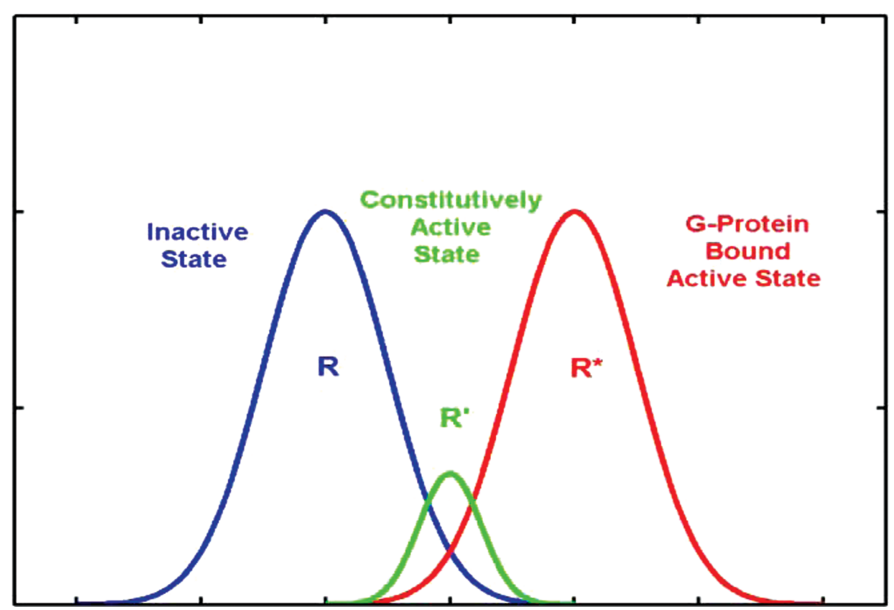

Figure 11.6 Functional ensemble view of GPCR conformations (adapted from ref. 53).

Kenakin provides a nice ensemble framework to think about multiple conformations of GPCRs. ${ }^{53}$ Let us call $\mathbf{R}$ the set or ensemble of conformations that correspond to the inactive state of a GPCR. Similarly, let us call $\mathbf{R} *$ the set of active conformations. GPCRs commonly show constitutive activity, which can have a phenotype distinct from an active state or an inactive state, so let us call $\mathbf{R}^{\prime}$ the set of conformations which are constitutively active. In general, the $\mathbf{R}$ and $\mathbf{R} *$ sets could overlap in the region of constitutive activity, so the functional ensemble picture of GPCR dynamics can be represented by Figure 11.6. Also, a constitutively active conformation from $\mathrm{R}^{\prime}$ can easily convert into an inactive (R) or an active $\left(\mathbf{R}^{*}\right)$ conformation depending on the conditions.

A picture is slowly emerging of the dynamic role played by these GPCR conformations in modulating and diversifying an extracellular signal inside the cell. Kenakin and Miller have aptly labeled these receptors as "shape-shifting" proteins to capture this conformational dynamism. ${ }^{54}$ In their words, these receptors are "pleiotropic" in terms of the multiple intracellular signaling cascades they can affect upon binding to an agonist (e.g. multiple G proteincoupled and $\beta$-arrestin coupled pathways). This opens the possibility of different agonists affecting the multiple intracellular signaling cascades differently, which appears to be the norm for these receptors. ${ }^{55}$ This not only turns the classical receptor theory for the relative efficacy of agonists on its head but also leads to diabolical ligand classifications as will be seen later in the chapter.

\subsubsection{Ligand or Mutation Stabilized Ensemble of GPCR Conformations}

Most GPCRs in the apo (ligand-free) form are capable of displaying constitutive (also called basal) activity. This state would correspond to the $\mathbf{R}^{\prime}$ set of conformations shown in Figure 11.6. GPCRs exist in this state ready to 
be converted to their active or inactive forms. They putatively go through a series of conformations during these conversions, where an agonist will stabilize conformations along the active pathways, and an inverse agonist will stabilize conformations moving towards the inactive form as shown in Figure 11.7. ${ }^{56}$ Even receptors like rhodopsin with no basal activity undergo transformations during activation from an inactive conformation to an active conformation through multiple intermediates. ${ }^{57}$

The Kobilka group has shown that in the presence of a single ligand (norepinephrine), the $\beta 2$ adrenergic receptor can exist in multiple conformational states ${ }^{51,58}$ as shown in Figure 11.8. They have also linked different ligands to specific conformational switches for the $\beta 2$ adrenergic receptor ${ }^{59}$ and have very recently shown conformational coupling between the extracellular surface (ECS) and orthosteric binding site in the TM region that can stabilize different conformations of a GPCR. ${ }^{60}$ Recent computational studies
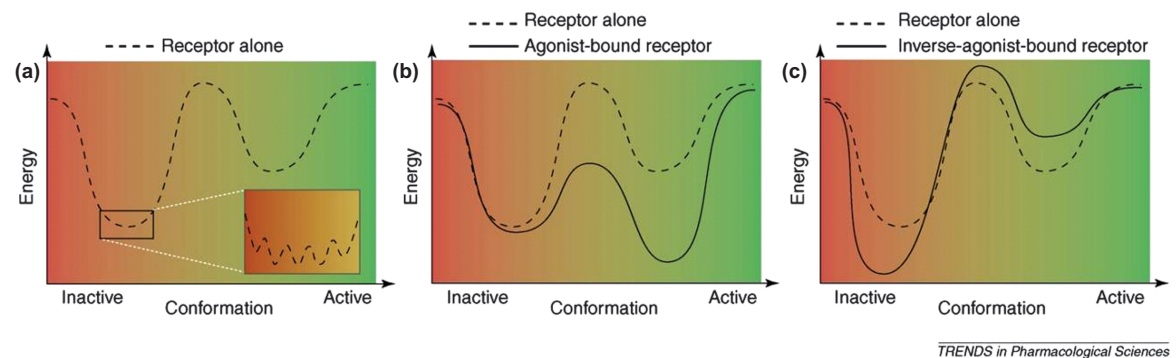

Figure 11.7 Energy landscape of the inactive and active GPCR conformations. Reprinted with permission from Elsevier from Kobilka and Deupi, Trends Pharmacol. Sci., 28(8), 397-406. Copyright 2007.

A

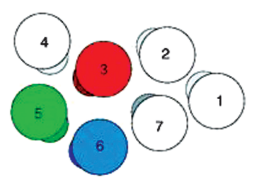

B

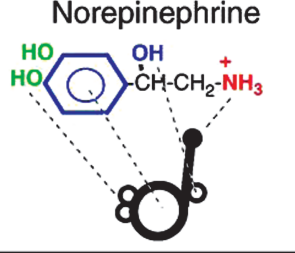

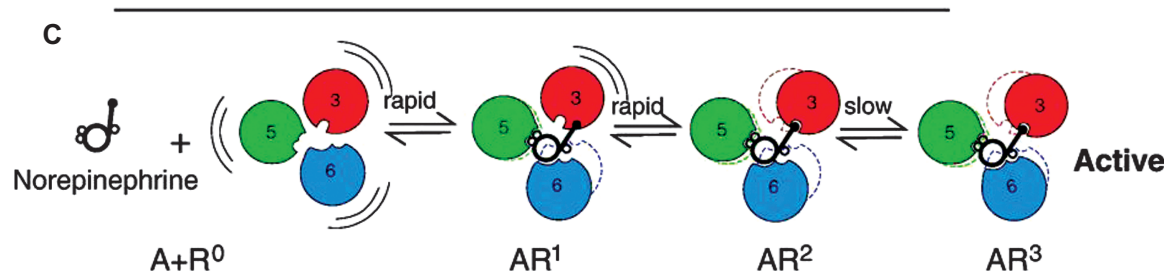

Figure 11.8 Sequential binding of norepinephrine to multiple $\beta 2$ adrenergic receptor conformations. Adapted from J. Biol. Chem., 279, 686. Copyright 2004, American Society for Biochemistry and Molecular Biology, Inc. 
have been inspired by these experiments and starting from inactive structures (from crystals) provided a more detailed view of these ligand-stabilized conformations, ${ }^{61,62}$ which is consistent with experimental observations. These observations of ligand-stabilized conformational states of a GPCR open the possibility of different agonists stabilizing different active conformations with different functional roles as will be seen later.

The seven-helix topology of GPCRs also enables a mutation to have a significant effect on their conformations accessible under physiological conditions. The Kendall group was able to show using two mutations at the same position in the cannabinoid $\mathrm{CB} 1$ receptor that the receptor can be switched to the constitutively active form $\left(\mathbf{R}^{*}\right)$ or into the inactive form $(\mathbf{R})$ with no basal activity. ${ }^{52}$ They found that the T210I mutant converts the CB1 receptor into a more fully constitutively active state $\left(\mathbf{R}^{*}\right)$, showing enhanced binding efficacy for agonists and diminished binding efficacy for inverse agonists. They also find that the T210A mutant is constitutively inactive, i.e. it converts the $\mathrm{CB} 1$ receptor into a fully inactive state $(\mathbf{R})$, which shows enhanced binding efficacy for inverse agonists and weaker binding for agonists. This strongly suggests that even in the absence of any ligand, GPCRs are capable of major conformational changes with potentially different functional outcomes.

We have recently predicted an ensemble of structures (conformations) for the CCR5 chemokine receptor that is a co-receptor for HIV entry using our GEnSeMBLE method for the efficient optimization of helix tilt angles $(\theta, \phi)$ and rotation angle $(\eta)$ that were defined in Figure 11.2A. The top lowest energy predicted conformations are shown in Table 11.2 and how they differ from each other in terms of structure and energies.

We have also discovered that mutations can have significant impact on the ensemble of conformations accessible by the apo proteins. Table 11.3 and Figure 11.8 illustrate this using CCR5 mutants as examples. The W86A CCR5 mutant conformations show that the wild-type \#30 (wt30) structure now ranks at \#4 (Table 11.3). Also, Figure 11.9 shows that this Trp86 residue has a steric clash with helix 7 in the wild-type structure, which disappears in the W86A mutant increasing its stability. The table also shows that for the $\mathrm{A} 90 \mathrm{H}$ mutant, the wt1 structure disappears from the top conformations, in this case due to steric clash of His90 with helix 7.

This phenomenon is not fully appreciated for GPCRs, but has the potential to explain the origin of the effect of mutations on ligand binding, whether the mutation affected the protein structure or directly affected ligand binding. We have docked multiple ligands to wild-type CCR5 and mutant ensemble of conformations. The results for binding of Maraviroc (the only drug in the market aimed at CCR5) are shown in Table 11.4, which shows the different protein conformations preferred by the wt and mutant proteins in apo and Maraviroc bound forms. We also find that different ligands (Maraviroc, PF-232798, Aplaviroc) prefer different CCR5 conformations, which is consistent with observations that these ligands bind to the same site but show different interaction profiles in mutagenesis studies ${ }^{63,64}$ and in effects on antibody binding. ${ }^{65}$ 


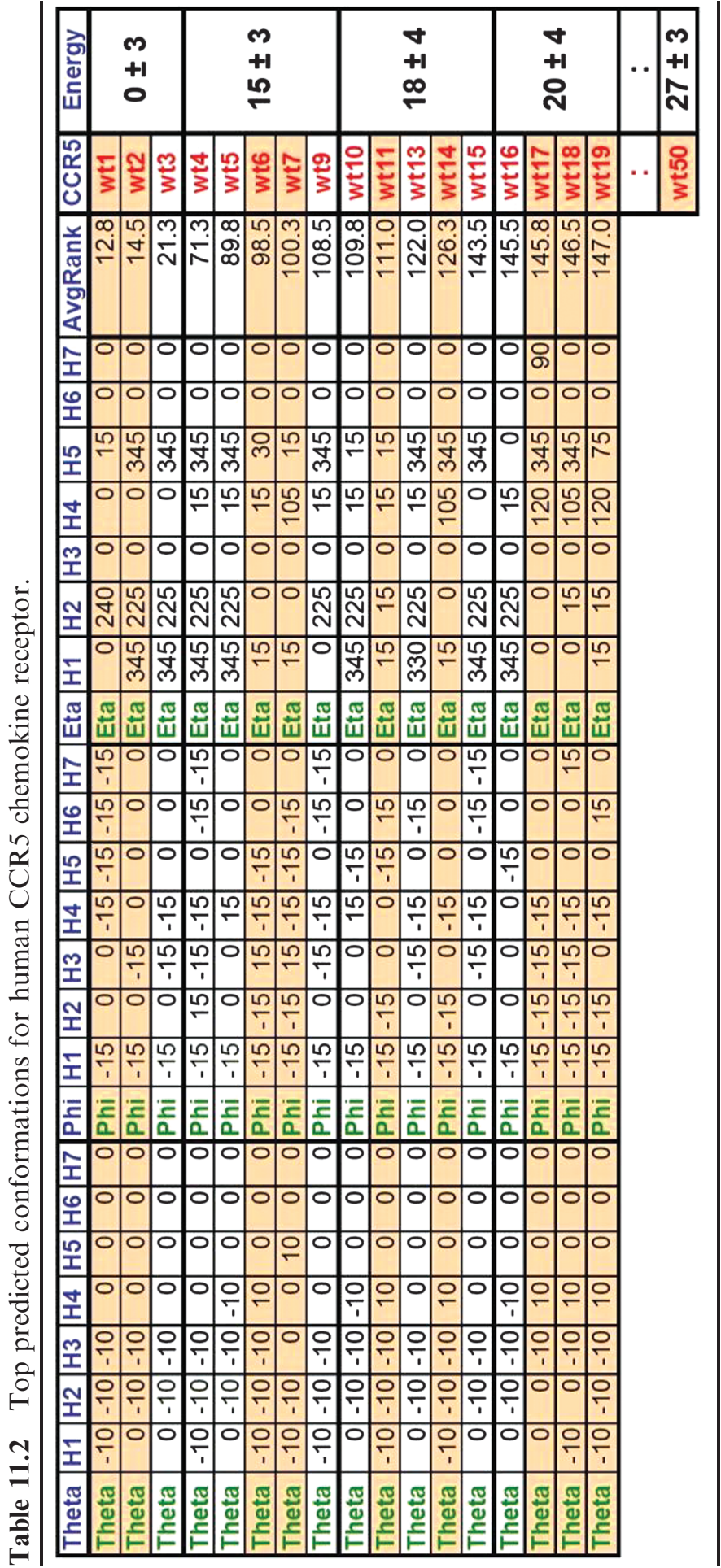


Table 11.3 Reordering of CCR5 wild-type conformations for mutants W86A, A90H and T105A.

\begin{tabular}{|c|c|}
\hline W86A & AvgRank \\
\hline wt1 & 8.0 \\
\hline wt3 & 11.8 \\
\hline wt2 & 15.0 \\
\hline wt30 & 16.0 \\
\hline wt6 & 20.8 \\
\hline wt17 & 26.0 \\
\hline wt7 & 28.3 \\
\hline wt8 & 29.3 \\
\hline wt4 & 30.0 \\
\hline wt18 & 30.3 \\
\hline wt11 & 30.3 \\
\hline wt48 & 31.0 \\
\hline wt20 & 31.3 \\
\hline wt12 & 31.3 \\
\hline wt19 & 34.8 \\
\hline wt14 & 34.8 \\
\hline wt9 & 35.8 \\
\hline wt27 & 36.0 \\
\hline wt34 & 37.0 \\
\hline wt13 & 38.0 \\
\hline
\end{tabular}

Wildtype \#30 becomes W86A\#4

\begin{tabular}{|c|c|}
\hline A90H & AvgRank \\
\hline wt3 & 3.0 \\
\hline wt2 & 6.0 \\
\hline wt4 & 15.3 \\
\hline wt5 & 16.3 \\
\hline wt13 & 18.5 \\
\hline wt10 & 21.0 \\
\hline wt9 & 22.5 \\
\hline wt27 & 22.8 \\
\hline wt14 & 23.8 \\
\hline wt32 & 24.8 \\
\hline wt64 & 25.0 \\
\hline wt15 & 25.0 \\
\hline wt16 & 25.8 \\
\hline wt26 & 27.0 \\
\hline wt28 & 27.5 \\
\hline wt31 & 28.5 \\
\hline wt17 & 29.3 \\
\hline wt33 & 30.3 \\
\hline wt11 & 32.0 \\
\hline wt74 & 32.5 \\
\hline
\end{tabular}

wt1 not in top 20?

\begin{tabular}{|c|c|}
\hline T105A & AvgRank \\
\hline wt1 & 4.0 \\
\hline$w t 3$ & 7.8 \\
\hline$w t 2$ & 10.0 \\
\hline$w t 11$ & 28.3 \\
\hline$w t 9$ & 28.8 \\
\hline$w t 12$ & 29.3 \\
\hline$w t 18$ & 30.5 \\
\hline wt4 & 30.5 \\
\hline wt27 & 30.8 \\
\hline wt6 & 31.0 \\
\hline wt7 & 31.3 \\
\hline wt20 & 31.5 \\
\hline wt8 & 31.8 \\
\hline wt14 & 31.8 \\
\hline wt13 & 34.0 \\
\hline wt15 & 36.0 \\
\hline wt5 & 36.0 \\
\hline wt64 & 37.3 \\
\hline wt32 & 38.0 \\
\hline wt30 & 42.5 \\
\hline
\end{tabular}

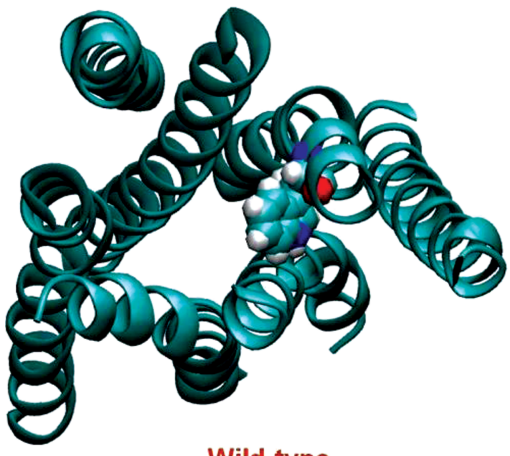

Wild-type

(Trp86 bumps into TM7)

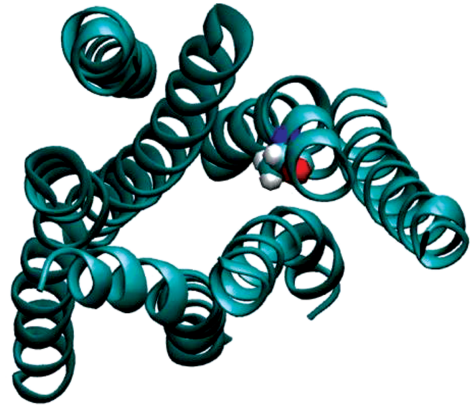

W86A

(Ala86 doesn't have the bump)

Figure 11.9 Structural basis for mutation effect on conformations (see text).

Next, we will describe the known intracellular consequences of GPCR activation and how the sensing of a single signal by the receptor can turn on a very wide range of intracellular signaling cascades. 
Table 11.4 Reordering of wild-type CCR5 conformations for various mutants and the conformation preferred by Maraviroc for each of the mutants.

\begin{tabular}{|c|c|c|}
\hline \multirow{2}{*}{ Mutations } & \multicolumn{2}{|c|}{ CCR5 Confs } \\
\cline { 2 - 4 } & Apo & Mara \\
\hline Wild-Type & wt1 & wt7 \\
\hline F109Y & wt3 & wt5 \\
\hline F112A & wt1 & wt5 \\
\hline Q194A & wt1 & wt5 \\
\hline Y251F & wt1 & wt2 \\
\hline D276A & wt1 & wt2 \\
\hline Q277A & wt1 & wt5 \\
\hline W86A & wt1 & wt3 \\
\hline A90H & wt3 & wt10 \\
\hline T105A & wt1 & wt2 \\
\hline Y108A & wt3 & wt2 \\
\hline F109A & wt1 & wt5 \\
\hline I198A & wt1 & wt5 \\
\hline Y251A & wt2 & wt3 \\
\hline Q280A & wt3 & wt5 \\
\hline E283A & wt3 & wt3 \\
\hline
\end{tabular}

\subsubsection{Intracellular Signal Diversification and GPCR Regulation}

GPCR activation can lead to a diverse set of signaling events inside the cell. So, these receptors can be called pleiotropic. These signaling events are mainly transduced through multiple $G$ proteins and arrestins (Figure 11.10). GPCR coupling to G proteins (which gave these receptors the name "GPCR") enables signal diversification inside the cell as these $G$ proteins control a spectrum of downstream signaling pathways. These pathways respond within seconds to minutes of the sensing of the signal by the GPCR. Arrestins appear to be playing a dual role of signal diversification as well as GPCR regulation (receptor desensitization and internalization). Arrestin-mediated signaling responses have a slower onset and are sustained over minutes to hours. As will be shown later, the ability of a single receptor to activate multiple pathways inside the cell can have important therapeutic consequences, as the G proteinmediated pathway may have a therapeutic benefit and the arrestin-mediated pathway may have unintended side-effects or vice versa. Next we will briefly describe the G protein-mediated and arrestin-mediated pathways and how their actions define GPCR function.

11.3.2.3.1 G Protein Mediated Signal Diversification. GPCRs got their name due to coupling to $G$ proteins, which are heterotrimeric proteins with 


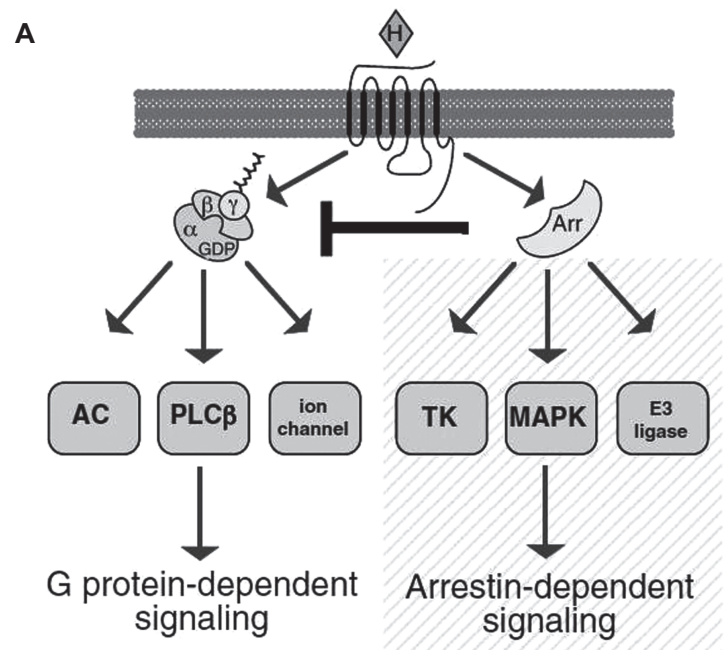

B

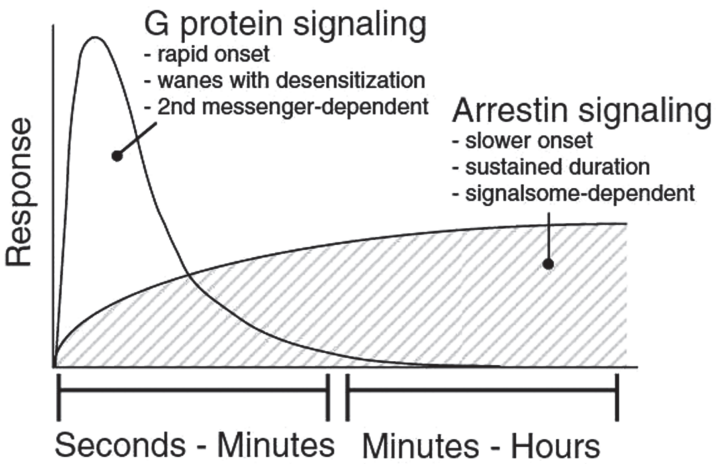

Figure 11.10 A G protein and arrestin-dependent signaling pathways. B Difference in response times from different pathways. Reproduced with permission from American Society for Pharmacology and Experimental Therapeutics from Luttrell and Gesty-Palmer, Pharmacol. Rev., 62, 305-330. Copyright 2010.

$\mathrm{G} \alpha, \mathrm{G} \beta$ and $\mathrm{G} \gamma$ subunits. The $\mathrm{G} \alpha$ subunit is made up of a ras-like GTPase domain and a helical domain. The guanine nucleotide GDP is sandwiched between these two domains. There are 23 known $\mathrm{G} \alpha$ proteins that can be assigned to four major classes represented by $\mathrm{G} \alpha_{\mathrm{s}}, \mathrm{G} \alpha_{\mathrm{i} / \mathrm{o}}, \mathrm{G} \alpha_{\mathrm{q} / 11}$ and $\mathrm{G} \alpha_{12 / 13}$ proteins. The $\mathrm{G} \beta$ subunit forms a bridge between $\mathrm{G} \alpha$ and $\mathrm{G} \gamma$ subunits, which don't appear to have a close contact as shown in Figure 11.11. There are 5 known human $\mathrm{G} \beta$ and 12 human $\mathrm{G} \gamma$ subunits, which can lead to a large number of possible $G \beta \gamma$ dimers that are coupled to many downstream signaling pathways ${ }^{66,67}$ as will be discussed below.

The inactive G protein exists in the heterotrimeric form bound to ADP. The activation of a GPCR upon agonist binding leads to a series of conformational 


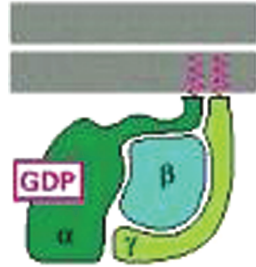

(A)

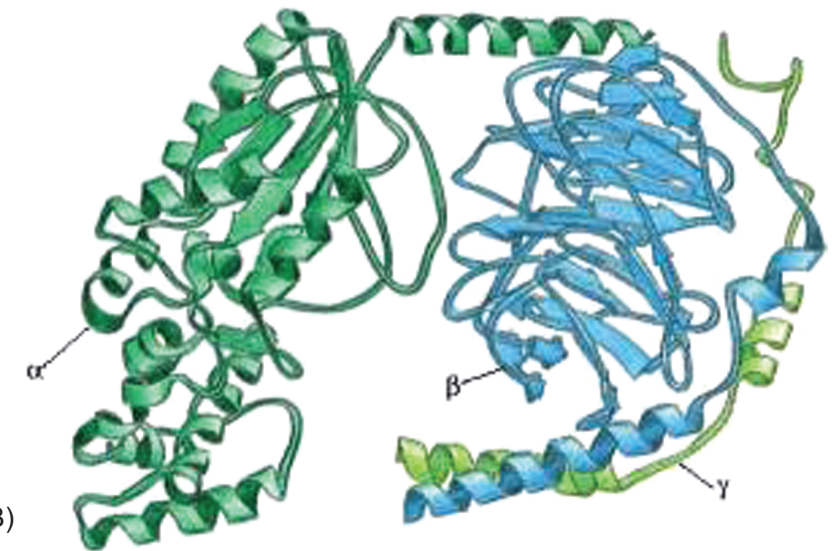

Figure 11.11 The architecture of the heterotrimeric $G$ protein.

changes, which recruit binding to the $\mathrm{G}$ protein using the receptor's intracellular loops and the C-terminus residues interacting mainly with the $\mathrm{G} \alpha$ subunit. This association leads to the dissociation of the $G$ protein into the $G \alpha$ subunit (which undergoes a conformational change coupled to the displacement of ADP by ATP) and the heterodimer complex G $\beta \gamma$ as shown in the left panel of Figure 11.12 that doesn't undergo any major conformational change. Each of these units, the $G \alpha$ subunit and the G $\beta \gamma$ complex, activates specific signaling pathways inside the cell. A recent crystal structure of Opsin (ligand-free form of rhodopsin) bound to the carboxy terminus of the $\mathrm{G} \alpha$ subunit $^{28}$ has shed light on the detailed molecular level contacts present between GPCRs and G proteins as shown in the right panels of Figure 11.12, which highlights through the middle panel the conformational change necessary upon activation to accommodate binding to the $\mathrm{G} \alpha$ subunit.

The $\mathrm{G} \alpha$ subunit controls many downstream signaling cascades through different effector molecules. The $\mathrm{G} \alpha_{\mathrm{s}}$ class of proteins stimulate adenylyl cyclase (AC), which converts ATP into cyclic AMP (cAMP), whereas G $\alpha_{i}$ class inhibits $\mathrm{AC}$ thereby opposing the effect of $\mathrm{G} \alpha_{\mathrm{s}}$ class. This suggests how a single GPCR activated by the same agonist can have opposite effects inside the cell just by coupling to different $G$ proteins. The $G \alpha_{\mathrm{q}}$ class of subunits activate phospho-inositide-specific phospholipase C (PI-PLC) isoenzymes, which generate second messengers inositol 1,4,5-triphosphate (IP3) and diacylglycerol (DAG). IP3 activates $\mathrm{Ca}^{2+}$ channels leading to the increase in calcium concentration, which facilitates protein kinase $\mathrm{C}$ (PKC) activation by DAG. Besides this critical function that controls many cellular functions through phosphorylation events caused by PKC, DAG also acts as a source of prostaglandins and as a precursor of the endocannabinoid 2-arachidonoylglycerol among its other functions.

The G $\beta \gamma$ complex activates its own set of effectors and the first ones identified were $\mathrm{G}$ protein-regulated inward-rectifier $\mathrm{K}^{+}$channels (GIRKs). 


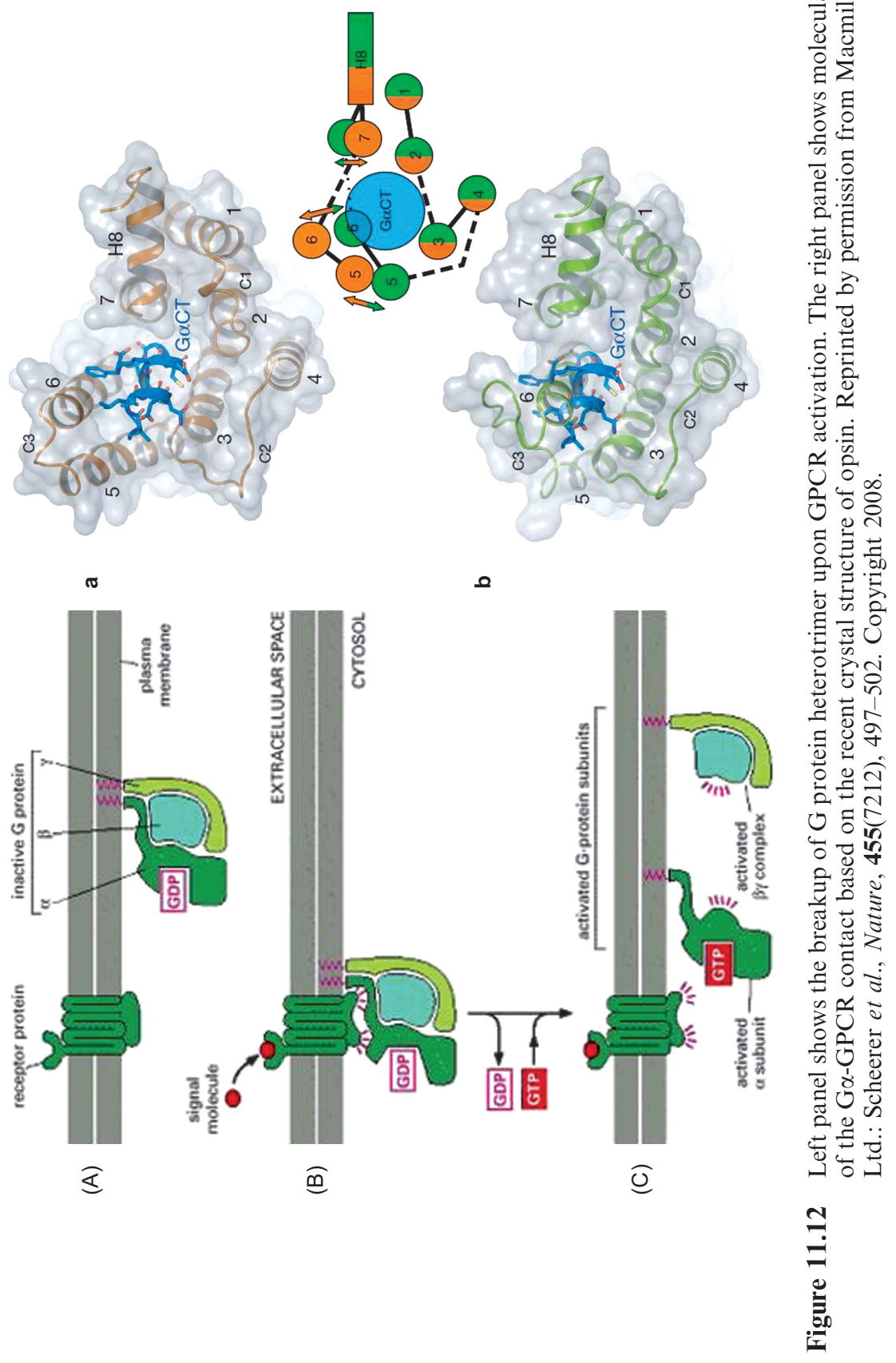

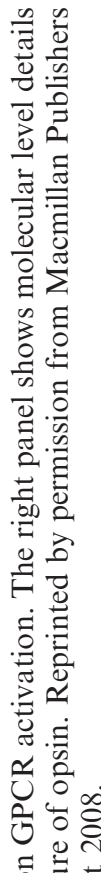

를 몰

के की

西? ट्रें 矛合 ป $\Xi \frac{1}{2}$ 으ำ 实 䓀 ป స

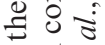
象 。 की बญे 牙艺 ב 
They stimulate many kinases like extracellular signal-regulated kinases (ERKs), c-Jun N-terminal kinases (JNKs) and p38 mitogen-activated protein kinases (MAPKs). The G $\beta \gamma$ complex can directly modulate $\mathrm{Ca}^{2+}$ channels and also upregulate or downregulate AC as well as PLC- $\beta$ activity. This range of effects allows the G $\beta \gamma$ complex to stimulate multiple signaling pathways inside the cell and to cross-talk with pathways activated by the $\mathrm{G} \alpha$ subunit as well. The G $\beta \gamma$ complex also recruits G protein-receptor kinases (GRK2 and GRK3) to the plasma membrane, where they phosphorylate agonist-bound GPCR conformations at the Ser and Thr positions in the intracellular loops or the Cterminus, preparing the receptor for subsequent binding to arrestins for further signaling and desensitization. However, this phosphorylation is not necessary in some cases to enable arrestin binding.

The intracellular signaling via $\mathrm{G}$ proteins can diversify depending on the type of coupling between GPCRs and G proteins. ${ }^{69}$ Four scenarios arise (as shown in Figure 11.13):

a) An agonist activates different GPCR subtypes that display highly specific $\mathrm{G}$ protein couplings, which as will be shown below can lead to very different downstream responses. This is most commonly observed in biogenic amine receptors for which multiple subtypes exist, e.g. there are nine adrenergic receptors, five dopamine receptors and four histamine receptors (Figure 11.13A).

b) An agonist activates a single GPCR, which activates the G protein and initiates multiple signaling cascades inside the cell. As mentioned above, upon activation $G$ protein breaks up into a $G \alpha$ subunit and $G \beta \gamma$ complex, both of which can promote signaling via the same effectors, or acting antagonistically, or cooperatively modulating one signaling

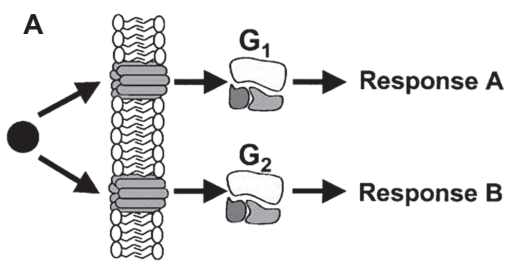

B
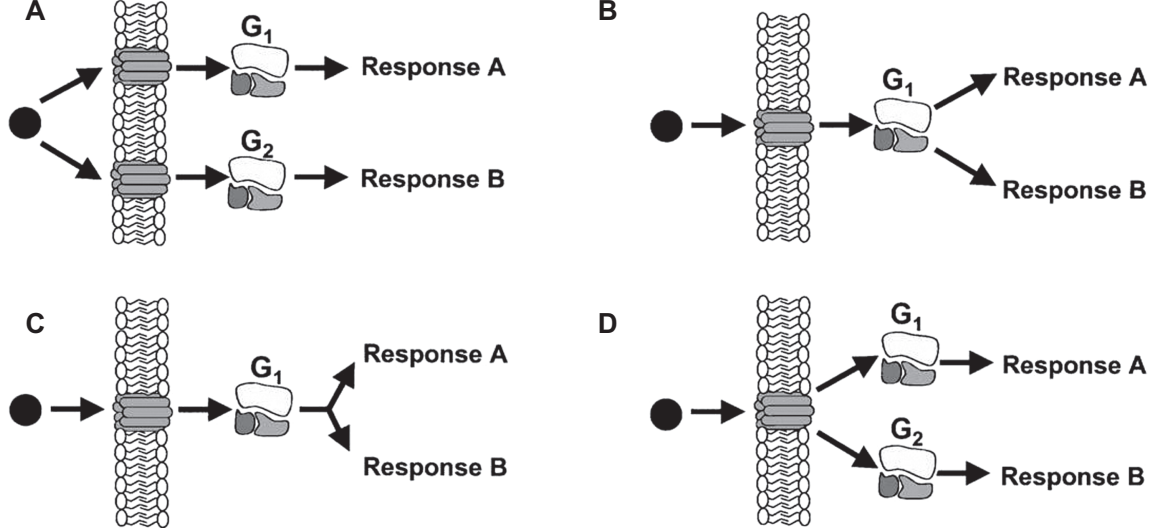

Figure 11.13 Different mechanisms of intracellular signaling divergence. Reprinted with permission from Elsevier from Pharmacol. Therap., 99(1), 25-44. Copyright 2003. 
pathway, or via different effectors leading to multiple signaling pathways (Figure 11.13B).

c) Agonist binding to GPCR activates a $\mathrm{G}$ protein leading to a single signaling pathway inside the cell, which diverges downstream (Figure 11.13C).

d) GPCR activation allows it to couple to multiple $G$ proteins inside the cell, which can activate many signaling pathways (Figure 11.13D).

These different scenarios are enabled due to conformational flexibility of activated GPCRs, which allows them a range of controls over intracellular signaling pathways. Next, we will describe the interaction of GPCRs with arrestins, which is another mode by which they activate various pathways inside the cell.

11.3.2.3.2 Arrestin Mediated Signaling and GPCR Regulation. $\beta$-Arrestins were originally thought to be only responsible for desensitization of activated GPCRs followed by internalization and recycling or degradation. As will be shown below, this view has changed dramatically and arrestins can activate multiple signaling pathways independent of any $G$ protein induced signaling. The arrestin family comprises four proteins in vertebrates: visual arrestins (arrestins 1 and 4) as well as $\beta$-arrestins 1 and 2 (arrestins 2 and 3). ${ }^{68}$

Desensitization of GPCRs (or loss of $\mathrm{G}$ protein-coupled response in the presence of an agonist) has been reviewed elsewhere ${ }^{70}$ and lucidly depicted (along with the endocytosis mechanisms) in Figure 11.14. A succinct picture of classical desensitization involves receptor phosphorylation by second messenger-stimulated protein kinases (like PKA and PKC) or by specific GRKs followed by arrestin binding to the intracellular receptor site that sterically hinders any further $G$ protein coupling. In addition, two new roles have emerged for $\beta$-arrestins in desensitization: a) degradation of second messenger molecules like cAMP ${ }^{71}$ and diacylglycerol (DAG); ${ }^{72}$ and b) limiting or reducing the generation of second messenger molecules as shown in a study ${ }^{73}$ that $\beta$-arrestin promotes the switching from $G_{s}$ to $G_{i}$ coupling, which further limits the production of cAMP. The class A GPCRs show greater affinity for $\beta$-arrestin 2, whereas class B GPCRs bind to $\beta$-arrestin 1 and 2 with equal affinity.

The receptor internalization (or endocytosis) is mediated by $\beta$-arrestin binding to clathrin via the adaptor protein AP-2, which targets the GPCRs to clathrin-coated pits (CCPs). These CCPs are pinched off from the plasma membrane by a large GTPase dynamin (see Figure 11.14) into endosomal vesicles, which either recycle the receptor to the plasma membrane or take the receptor through degradation pathways. In the vesicle, the agonists are released from the receptor due to the acidic environment of the vesicle and the receptor is dephosphorylated in preparation for recycling back to the plasma membrane (or resensitization).

One of the important functions of GPCRs is the regulation of cell proliferation and differentiation, via the activation of mitogenic pathways. The 


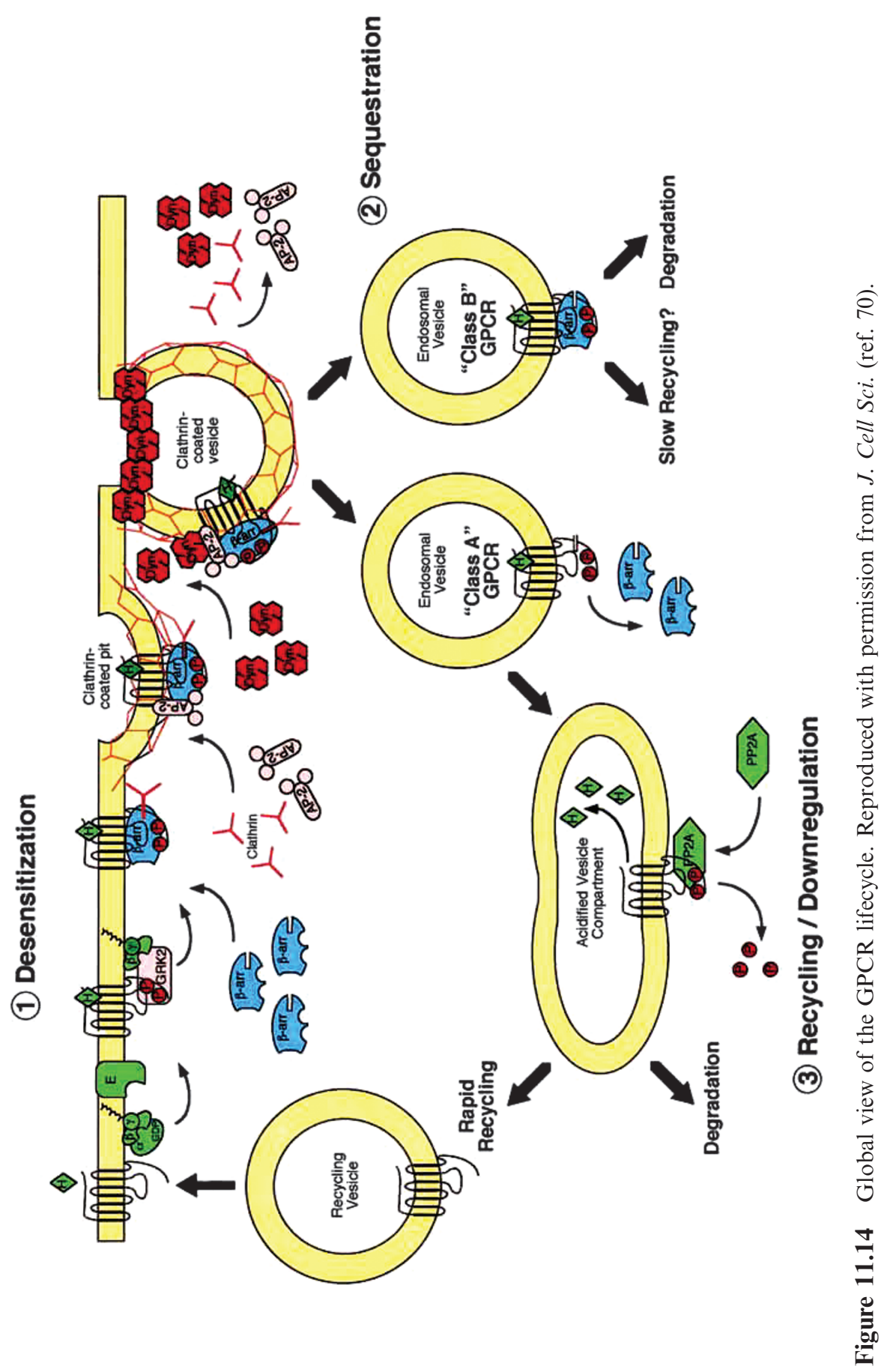


$\beta$-arrestins play a vital role in the promotion of the formation of multi-protein signaling complexes with ERK and various tyrosine kinases involved in these mitogenic pathways independent of $G$ protein activation. Specific GRKs can induce receptor phosphorylation that can lead to agonist-stimulated ERK activation in the absence of $\mathrm{G}$ protein activation. The angiotensin II activation of angiotensin II type 1 receptor (AT1R) with a mutation in the conserved DRY motif (at the intracellular end of TM3) doesn't cause any G protein-mediated signaling, but maintains $\beta$-arrestin recruitment and ERK activation. ${ }^{74,75}$ In addition, the use of siRNA against $\beta$-arrestin 2 blocks the angiotensinstimulated ERK activation. ${ }^{76}$ This elucidates that GPCRs can signal exclusively through $\beta$-arrestins even in the absence of $G$ protein coupling. This appears to be a more general feature of GPCR signaling as the same has been shown for another rhodopsin family receptor $\beta 2 \mathrm{AR}^{77}$ and also for a secretin family receptor type $1 \mathrm{PTH} / \mathrm{PTH}$-related peptide receptor. ${ }^{78}$ A general view of this biased signaling is shown in Figure 11.15, where either a ligand could bias the receptor towards $\beta$-arrestin pathway, or a protein might be innately biased towards $\beta$-arrestin pathway as shown recently for the CXCR7 receptor. ${ }^{79}$

\subsubsection{GPCR Dimers and Interaction with Other Proteins}

All prior discussion in the chapter implicitly assumed that GPCRs couple to $\mathrm{G}$ proteins as monomers. Any functional association of a GPCR with its copy, or a different GPCR, or another protein only increases the GPCR repertoire of signaling mechanisms for selective functional control within the cell. Early evidence of GPCR dimerization was observed in recombinant cell systems that over-expressed these receptors, which raised doubts about their functional significance. ${ }^{80}$ There is now strong functional evidence based on experiments

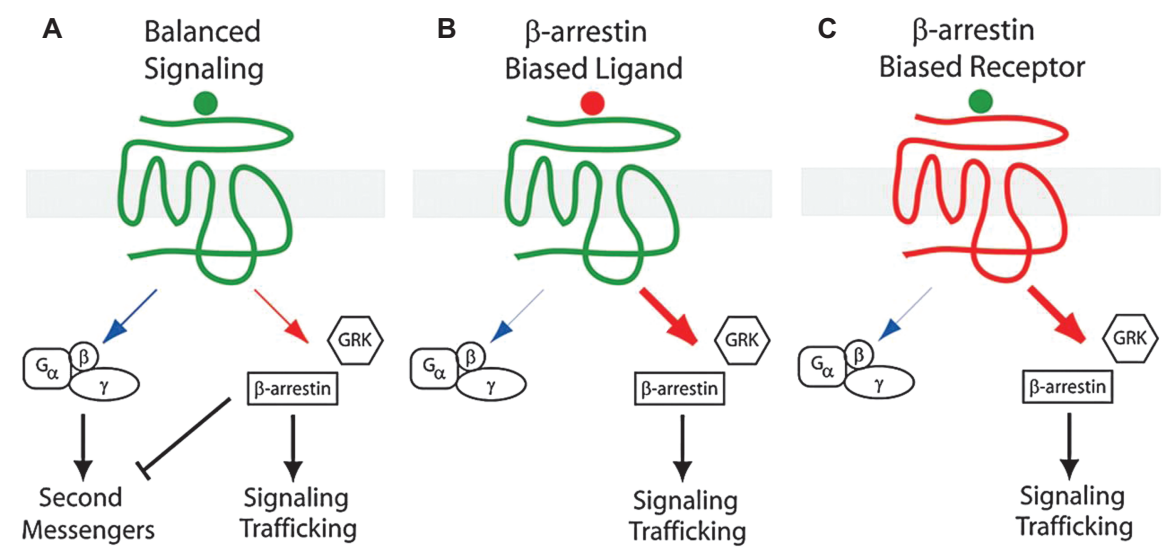

Figure 11.15 Balanced and biased signaling by GPCRs. Reproduced with permission from Rajagopal et al., Proc. Nat. Acad. Sci. U. S. A., 107(2), 628. Copyright 2010. 
involving native tissues that some GPCRs form homodimers and some form heterodimers in their functional forms.

A GPCR class $C$ receptor $m$ Glu5 has been shown to be a homodimer stabilized by a disulfide bridge in the extracellular domain. ${ }^{81}$ The extracellular VFT domain of mGlul receptor has been crystallized as a dimer with a disulfide bridge in different relative orientations of the monomers depending on the presence or absence of the agonist glutamate, ${ }^{82,83}$ suggesting a possible mechanism of activation involving the relative change in the orientation of the monomeric TM bundles in the homodimer. ${ }^{84}$

Another class C GPCR provided the first conclusive evidence of a functional heterodimer. The $\gamma$-aminobutyric acid-binding $\left(\mathrm{GABA}_{\mathrm{B}}\right)$ receptor is only functional as a heterodimer between $\mathrm{GABA}_{\mathrm{B} 1}$ and $\mathrm{GABA}_{\mathrm{B} 2}$ monomeric subunits, where each subunit plays a distinct role: $\mathrm{GABA}_{\mathrm{B} 1}$ subunit binds to the agonists and $\mathrm{GABA}_{\mathrm{B} 2}$ subunit couples to the $\mathrm{G}$ protein. ${ }^{85}$ Sweet and umami taste receptors provide other class $\mathrm{C}$ examples of heterodimerization. Of the three genes encoding these receptors (T1R1, T1R2 and T1R3), T1R2-T1R3 heterodimer results in a sweet receptor and the T1R1-T1R3 heterodimer results in an umami receptor. ${ }^{14}$ The three monomeric receptors don't display the functional behavior if they are expressed alone.

There is also plenty of evidence now in favor of the presence of functional homodimers and heterodimers in class A GPCRs. ${ }^{80}$ Here we will mention a more recent example involving the dopamine D2 receptor, which has been shown to form homodimers out of monomers in functionally different states; maximal activation was observed when one monomer was bound to the agonist (was active) and the other monomer was bound to an inverse agonist (was inactive). ${ }^{86}$

Interaction of GPCRs with other membrane and cytoplasmic proteins has been known for a while and has also been reviewed. ${ }^{87}$ The physiological implications of these interactions are slowly being uncovered as only some of these interactions have been amenable to detailed experimental investigations. A discussion of these interactions is beyond the scope of this chapter.

\subsubsection{Functional Control of GPCRs by Ligands}

As mentioned earlier, GPCRs are pleiotropic in terms of the multiple intracellular signaling cascades they can affect upon binding to an agonist through both $\mathrm{G}$ protein-coupled and $\beta$-arrestin coupled pathways. Experimentally, a single functional assay (usually by definition) cannot see all the signaling effects of a ligand. It is now evident that agonist-bound GPCRs exist in multiple distinct conformations, where each conformation can potentially activate a different signaling pathway. From a therapeutic perspective, this may not be desirable if, of the multiple signaling pathways activated by a drug-molecule, one pathway may be mainly responsible for the desirable therapeutic benefit and another may be causing unwanted side-effects. This opens at least two distinct possibilities of controlling the functional consequences of GPCR activation: biased agonists and allosteric modulators. Each of these possibilities will be 
described below along with a few representative examples. As will be seen, some of these ligands defy the classical definitions of agonists and antagonists.

\subsubsection{Biased Agonism}

Ligands and especially agonists can induce multiple GPCR conformations upon binding. As has been mentioned before, GPCRs interact with cytosolic G proteins and $\beta$-arrestins. Different ligands can induce a different ensemble of GPCR conformations, which will have a different range of interactions with $G$ proteins and/or $\beta$-arrestins and hence induce different intracellular signaling pathways in a ligand-dependent fashion. As shown in Figure 11.16, agonist

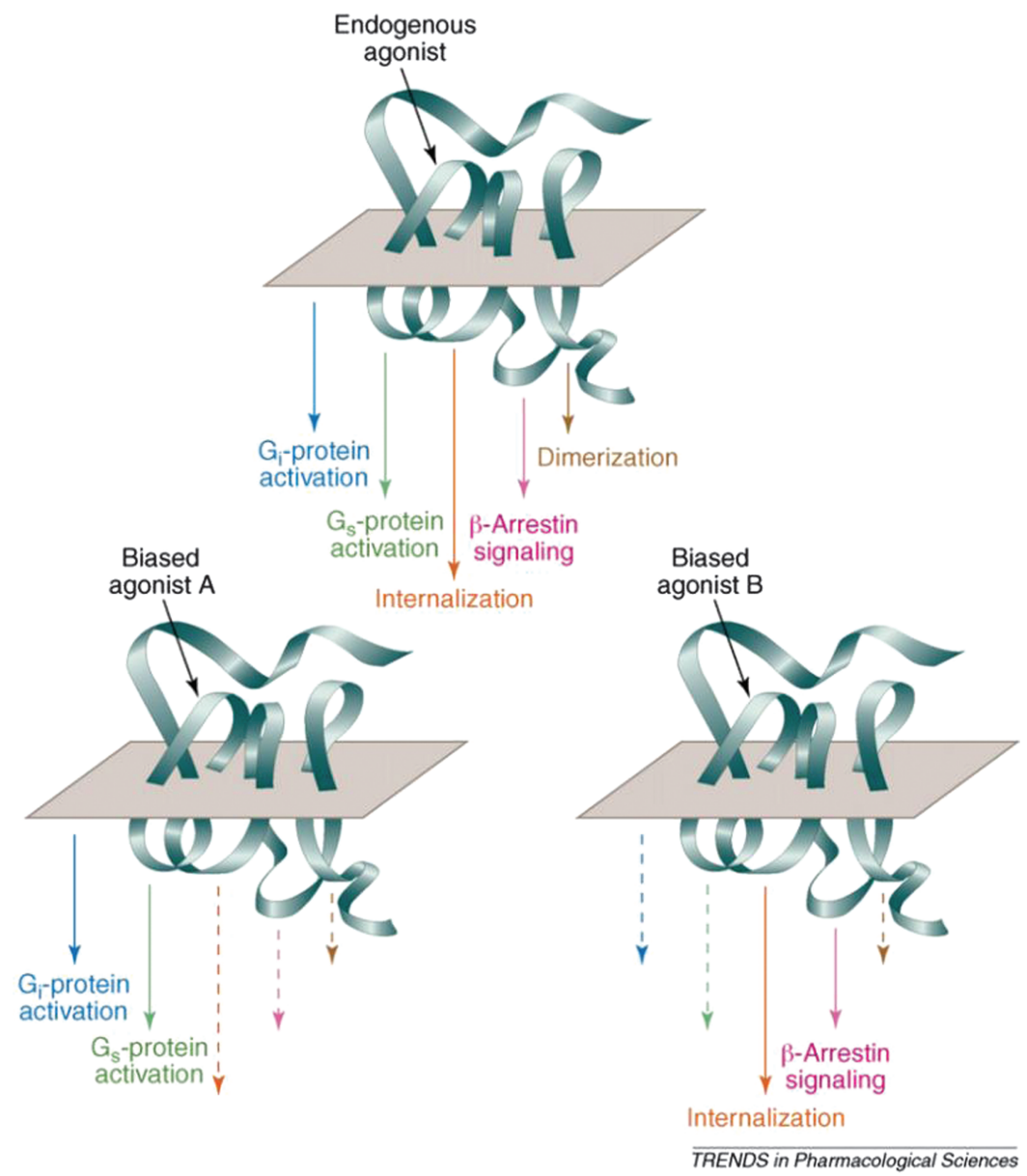

Figure 11.16 Multiple pathways that can be initiated by GPCR activation and biased by ligands. Reprinted with permission from Elsevier from Kenakin, Trends Pharmacol. Sci., 28(8) 407-415. Copyright 2007. 
binding to GPCRs can induce a cascade of processes, some through G protein coupling and some through $\beta$-arrestin coupling. As shown before, $\beta$-arrestin coupling can lead to specific signaling events and receptor endocytosis, which can further lead to either recycling of the receptor or its degradation. Figure 11.16 also shows the effect of biased agonists A and B that can either exist or be designed to activate a subset of possible signaling pathways.

Classically, the GPCR agonists have been characterized as such by their effect on $\mathrm{G}$ protein-coupled pathways. Experiments measuring $\beta$-arrestin signaling are becoming more commonplace so the effects of classical agonists need to be evaluated and should lead to a detailed characterization of these ligands. The parathyroid hormone (PTH), for example, can activate extracellular signal-related kinase using distinct $G$ protein-dependent and $G$ proteinindependent pathways. The PTH analogs, however, can use the same PTH receptor and separately use either $\mathrm{G}$ protein-dependent or -independent pathways. An example is that [Trp $\left.{ }^{1}\right] \mathrm{PTHrp}-(1-36)$ stimulates ERK1/2 via $\mathrm{G}$ protein pathway, whereas PTH-1A [[D-Trp12,Tyr34]PTH-(7-34)] stimulates the same via $\beta$-arrestin pathway in a $G$ protein-independent manner. ${ }^{78}$ This can have direct therapeutic consequences because PTH regulates calcium homeostasis as well as bone metabolism and utilization of $\beta$-arrestin 2 pathway is critical for this benefit, ${ }^{88}$ so $\beta$-arrestin biased PTH analogs mentioned above provide potentially improved therapy for osteoporosis. Another example is that nicotinic acid is therapeutically very beneficial as an anti-lypolytic agent (via $\mathrm{G}$ protein-mediated pathways), but causes cutaneous flushing as a major side-effect, which has been directly linked to the activation of $\beta$-arrestin 1 pathways. ${ }^{89}$ An analog of this molecule that doesn't affect the $G$ protein pathways but blocks the $\beta$-arrestin 1 pathways will be highly desirable. This also necessitates new characterization of classical agonists, e.g. a ligand that blocks $G$ protein pathways but uses $\beta$-arrestin pathways may have been classified before as an antagonist (or inverse agonist) but now should be more accurately described as a $\beta$-arrestin biased agonist.

These studies are also increasing our understanding of the relationship between various signaling pathways and previously unexplained side-effects of drug molecules. The use of knowledge about biased signaling during the drug design phase has the potential to generate multiple novel ways to control and hopefully cure many ailments with minimal side-effects.

\subsubsection{Allosteric Ligands and Signal Modulation}

Orthosteric ligands bind to GPCRs in regions that fully or partially overlap with that of the endogenous ligand(s), thereby sterically excluding the possibility of both occupying the GPCR at the same time. Allosteric ligands bind to GPCRs in regions that don't overlap with the endogenous ligand binding site, so both can occupy the receptor at the same time. This can have important signaling consequences because, as mentioned before, agonists induce an ensemble of GPCR conformations with a range of functional implications and allosteric ligands can dramatically modulate those conformations (e.g. by 
stabilizing a subset), which can lead to modulation of signaling and hence function. Allosterism provides a powerful natural tool for modulating signaling cascades, but not many natural modulators are known, probably because of the difficulty in identifying these ligands which are structurally dissimilar to endogenous ligands. One of the examples is the unnatural D-amino acid D-serine formed in the brain, which is a strong allosteric modulator of the $N$-methyl-D-aspartate (NMDA) receptor. ${ }^{90}$

An allosteric modulator can bind to a unique ensemble of GPCR conformations and have three modulatory effects on GPCR activation: allosteric antagonism (including allosteric inverse agonism), allosteric agonism and allosteric partial antagonism. In allosteric antagonism, the modulator stabilizes more inactive conformations or destabilizes more active conformations, resulting in the net reduction of GPCR activation relating signaling. These are usually referred to as negative allosteric modulators (NAMs). In allosteric agonism, two scenarios arise where the modulator either enhances the effect of the orthosteric agonist by stabilizing the more active receptor conformations or directly causes the GPCR activation in the absence of the orthosteric agonist. The former kind are referred to as positive allosteric modulators (PAMs), some of which are capable of directly agonizing the receptor in the absence of the orthosteric agonist. ${ }^{91}$ In allosteric partial antagonism, the modulator (also called biased antagonist) selectively blocks only a subset of the activation related pathways as shown in Figure 11.17. The figure shows that Postaglandin $\mathrm{D}_{2}$ normally activates $\mathrm{G}$ protein as well as $\beta$-arrestin pathways for its CRTH2 receptors, where both of these pathways can be blocked by an orthosteric

\section{Biased Antagonism}
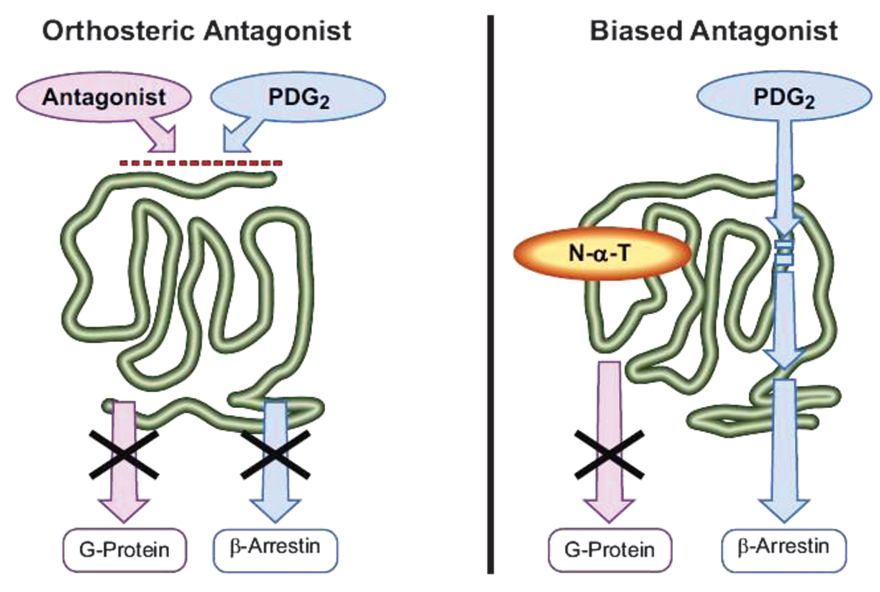

Figure 11.17 Biased antagonism, which can be called allosteric partial antagonism. Reproduced with permission from American Society for Pharmacology and Experimental Therapeutics from Kenakin and Miller, Pharmacol. Rev., 62, 265-304. Copyright 2010. 
antagonist. The biased antagonist $\mathrm{N}$ - $\alpha$-tosyltryptophan $(\mathrm{N}-\alpha-\mathrm{T})$ selectively blocks the $\mathrm{G}$ protein coupled pathways allowing the agonist PGD2 to continue to activate the $\beta$-arrestin pathways. ${ }^{92}$

There is plenty of evidence in favor of different allosteric modulators stabilizing a different subset of GPCR conformations, one being antibody binding profiles of Ab45531 and Ab45523 for the chemokine receptor CCR5, which is a coreceptor for HIV-1 entry. These antibody binding profiles differ in the presence of allosteric HIV-1 entry inhibitors like aplaviroc, TAK-779 and SCH-C. ${ }^{65}$

The muscarinic acetylcholine receptors (mAChRs) are class A GPCRs that provide a good pharmacological example for the need of selective allosteric modulators. ${ }^{93,94}$ There are five mAChR subtypes implicated in many physiological pathways. The acetylcholine binding site residues are highly conserved across these five receptor subtypes, making it difficult to design orthosteric agonists or antagonists. It has been shown that these receptors have one or two allosteric binding sites available for allosteric modulators to regulate the effect of orthosteric ligands. ${ }^{93}$ This provides for a general strategy for therapies targeting receptor subtype selectivity as the allosteric sites will be much less conserved than the orthosteric sites. In addition, a new class of modulators has emerged called "bitopic" that interact with both the allosteric and the orthosteric sites for self-modulation of their activity. ${ }^{95,96}$

The studies of these modulators really adds to the diverse ways in which GPCR conformational flexibility can be exploited for innate signaling as well as for therapeutic applications where target receptor subtype selectivity is highly desirable or activation of specific pathways causes undesirable side-effects.

\subsubsection{Challenges in GPCR Targeted Drug Design}

The lack of 3D structures for most human GPCRs (none were available until 2007) has led to the almost exclusive use of high-throughput screening (HTS) and virtual ligand screening (VLS) techniques in drug design, which do not require protein structures as input. Even though the number of compounds being screened by HTS and VLS techniques is higher than ever, the number of new approved drugs is on a decline. A large proportion (43\%) of drug candidates in clinical trials fail due to lack of efficacy and a significant one-third fail due to toxicity and side-effects. ${ }^{97}$ Some of these undesirable effects result from drugs hitting other GPCRs or even other subtypes of the same receptor, e.g. many dopamine D2 receptor agonists used for treating Parkinson's disease produce behavioral side-effects (e.g. compulsive gambling even in nongamblers $)^{98}$ because they actually bind with higher affinity to the dopamine D3 receptor which has been associated with emotion, reward and addiction. Such side-effects can be effectively minimized by designing D2 selective agonists, which requires atomic-level structures for both D2 and D3 receptors. As the theme of this chapter suggests that multiple GPCR conformations and their signaling consequences need to be accounted for, it is a daunting challenge. Concerted efforts are underway to crystallize a number of GPCRs with agonists, antagonists and inverse agonists, which will slowly provide valuable 
information. Parallel to these efforts, validated structure prediction methods that can generate an ensemble of GPCR structures that can be validated by mutagenesis as well as functional/binding assays and provide avenues for rational structure-based drug design are highly desirable.

Identification of the molecular basis of toxicity and other side-effects has been possible for a very few cases, some of which have been mentioned in this chapter. A recent pertinent example is the anti-obesity target, the cannabinoid CB1 receptor, which is a GPCR stimulated by cannabis, generating feelings of euphoria. Application of two promising anti-obesity CB1 antagonists, rimonabant (marketed, and then pulled from the European Union) and taranabant, resulted in adverse psychiatric symptoms like depression, anxiety and suicidal ideation. This has led many big pharmaceutical companies to abandon drug development programs on CB1 target, as it is not clear whether sideeffects are due to off-target interactions (i.e. not related to $\mathrm{CB} 1$ ) or due to $\mathrm{CB} 1$ antagonism.

This leads to two possible scenarios. Firstly, if the side-effects are confirmed to be off-target and it becomes known which receptors need to be avoided (hereafter called anti-targets), the structure-based rational drug design process can potentially use the structures of the target receptor and all the anti-target receptors to tailor the drug towards the target and away from the anti-targets, taking into account any information about signaling pathways that might be available. Secondly, if the side-effects are confirmed to be due to the target receptor, then it is most likely due to one of the multiple signaling pathways activated by the receptor. This will require a detailed therapeutic characterization of conformation-specific signaling pathways for the target receptor, so that biased agonists or allosteric modulators can be rationally designed using the therapeutically beneficial pathways.

Identification of off-targets for side-effects of drugs is an area of active research. A recent study looked at 3665 FDA approved and investigational drugs and their chemical similarities against 200,000 ligands that have been organized into families based on the proteins they target. ${ }^{99}$ This identified thousands of unanticipated off-targets for the approved drugs. Some of these are shown in Figure 11.18 using red arrows. Based on these predictions, 30 off-target associations were tested experimentally and 23 were confirmed, 5 of which were potent $(<100 \mathrm{nM})$. Key unknown associations uncovered were that transporter inhibitor Prozac antagonizes the $\beta 1$ adrenergic receptor, the ion-channel drug Vadilex inhibits the 5-hydroxytryptamine (5-HT) transporter and the enzyme inhibitor Rescriptor antagonizes the histamine $\mathrm{H}_{4}$ receptor. Prozac's adverse effects in terms of SSRI-discontinuation syndrome and sexual dysfunction can be explained by its $\beta 1$ blocking ability as $\beta$ blockers have those effects. Vadilex and Rescriptor are displaying polypharmacology as they are hitting very unrelated receptors, confirming that structurally diverse proteins can bind to structurally similar ligands. Such analysis needs to become routine to help minimize potential side-effects of the drugs being developed.

Experimental studies are uncovering new signaling mechanisms and computational studies can complement them through their use in rational drug 


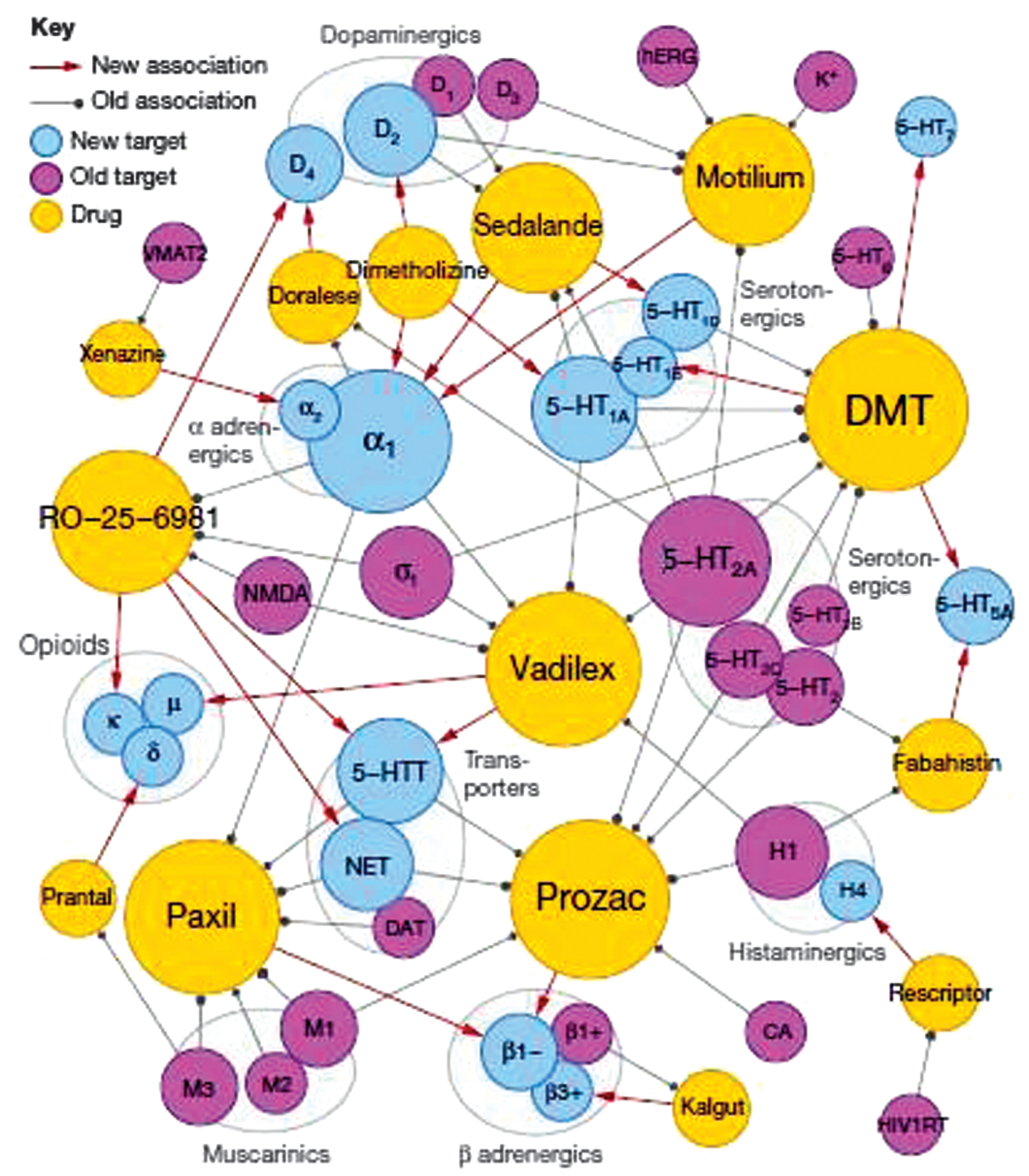

Figure 11.18 Off-target associations predicted for known drugs. Reprinted by permission from Macmillan Publishers Ltd.: Keiser et al., Nature, 462(7270), 175. Copyright 2009.

design approaches and in finding new off-targets. The complexity of GPCR signaling that underlies therapeutic applications requires a synergistic role for experimental and computational methods in producing novel therapies with minimal side-effects.

\subsection{Summary and Looking Ahead}

Significant efforts aimed at understanding the signaling from the protein level to the cellular level have uncovered multiple layers of complexity that would have been difficult to imagine even if the structures for all proteins involved were known. GPCRs have evolved into a nearly "perfect" receptor capable of activating and controlling multiple intracellular signals through its variety of 
conformational states, each with potentially different function. Ligands capable of stabilizing these distinct GPCR conformations have the potential to control GPCR signaling with high selectivity but with different functionality, as discussed in terms of biased agonists and allosteric modulators. As more signaling pathways are discovered downstream of GPCR activation, the need for such biased ligands is likely to increase.

Tremendous progress has been made over the last 20 years on GPCR function, broadening our perspective on the diverse capabilities of these receptors. In the last few years long-term GPCR crystallization efforts are finally bearing fruit, providing rapid progress in structural information. Complementing these experimental advances are dramatic improvements in the GPCR structure prediction methods aimed at identifying the multiple conformational states that appear sometimes to lead to different functions. As these predictions are validated with biological and X-ray experiments, we can expect new, more refined predictions on dynamical processes that are difficult to follow experimentally. We can expect that this will provide useful structural and functional data for GPCRs not yet crystallized and for orphan GPCRs whose functions are unknown. These theoretical and computational studies complemented with experiment are providing a basis for understanding the mechanism of activation so important for many diseases. The theory also has the potential to suggest ligands and/or mutations that would stabilize the active conformations of GPCRs so that they can be crystallized in their active form, the next "holy-grail" for GPCR structure determination.

We can expect the 3D structures and function of many GPCRs to be determined experimentally and computationally over the next decade or two, providing the potential for an enormous increase in our understanding of the various signaling processes in which they are involved.

The advances in our understanding of GPCR structure and function have resulted from recent breakthroughs in uncovering new signaling pathways. Molecular biology, GPCR crystallization and computational modeling have established a strong basis for proceeding to develop targeted subtype specific therapeutics that will likely help increase the efficacy while helping reduce the side-effects associated with current drugs ranging from Parkinson's to schizophrenia and from depression to obesity.

\section{Acknowledgements}

Our development of predictive methods for structures and function of GPCRs started around 1998 with an ARO-MURI grant oriented toward olfaction, a time when no 3D structures were available. The early advances were led by Dr. N. Vaidehi (now Professor of Immunology at City of Hope, Duarte, California) and Wely Floriano (now Associate Professor of Chemistry at Lakehead University, Ontario, Canada). Other important early contributions were made by Deqiang Zhang, Spencer Hall, Peter Freddolino, Eun Jung Choi, Georgios Zamanakos, Peter Kekenes-Huskey, Peter Freddolino, Yashar Kalani, Rene Trabanino, Victor Kam, Art Cho, John Wendel, P. Hummel, Peter Spijker; 
Joyce Yao-chun Peng, Youyong Li and Jiyoung Heo. More recently the team has been led by Ravinder Abrol, with major contributions from Jenelle Bray, Soo-Kyung Kim, Bartosz Trzaskowski, Adam Griffith, Caitlin Scott, Caglar Tanrikulu and Andrea Kirkpatrick. The funding sources that have allowed us to continue this project came from Sanofi Aventis, Boehringer-Ingelheim, Pfizer, Schering AG, PharmSelex and from NIH (R21-MH073910-01-A1, NIH 1 RO1 CA 112293-01, NIH 1R01 GM62523-01, NIH R01 AI40567).

\section{References}

1. J. T. Hancock, in Cell Signalling, Oxford University Press, Oxford, 3rd edn, 2010, p. 341.

2. G. Krauss, in Biochemistry of Signal Transduction and Regulation, WileyVCH, Weinheim, 3rd edn, 2003, p. 541.

3. J. Nelson, in Structure and Function in Cell Signalling, John Wiley \& Sons, Chichester, 2008, p. 389.

4. J. J. Allen, L. M. Mathger, A. Barbosa, K. C. Buresch, E. Sogin, J. Schwartz, C. Chubb and R. T. Hanlon, Proc. Royal Soc. B-Biol. Sci., 2010, 277(1684), 1031.

5. E. J. Kelman, D. Osorio and R. J. Baddeley, J. Exp. Biol., 2008, 211(11), 1757.

6. B. Alberts, in Molecular Biology of the Cell, Garland Science, New York, 4th edn, 2002, p. 1548.

7. D. Purves, Neuroscience, Sinauer, Sunderland, 4th edn, 2008, p. 857.

8. D. R. Robinson, Y. M. Wu and S. F. Lin, Oncogene, 2000, 19(49), 5548.

9. K. Lundstrom, Curr. Protein Pept. Sci., 2006, 7(5), 465.

10. J. P. Overington, B. Al-Lazikani and A. L. Hopkins, Nat. Rev. Drug Discov., 2006, 5(12), 993.

11. M. C. Lagerstrom and H. B. Schioth, Nat. Rev. Drug Discov., 2008, 7(4), 339.

12. T. Bartfai, Nat. Rev. Drug Discov., 2004, 3(7), 574.

13. R. Fredriksson, M. C. Lagerström, L. G. Lundin and H. B. Schiöth, Mol. Pharmacol., 2003, 63(6), 1256.

14. G. Q. Zhao, Y. Zhang, M. A. Hoon, J. Chandrashekar, I. Erlenbach, N. J. P. Ryba and C. S. Zuker, Cell, 2003, 115(3), 255.

15. J. Chandrashekar, K. L. Mueller, M. A. Hoon, E. Adler, L. Feng, W. Guo, C. S. Zuker and N. J. P. Ryba, Cell, 2000, 100(6), 703.

16. M. Behrens and W. Meyerhof, Cell. Mol. Life Sci., 2006, 63(13), 1501.

17. E. Rozengurt, Am. J. Physiol. Gastrointest. Liver Physiol., 2006, 291(2), G171.

18. S. Z. Hao, C. Sternini and H. Raybould, Gastroenterol., 2007, 132(4), A37.

19. T. Okada, M. Sugihara, A. N. Bondar, M. Elstner, P. Entel and V. Buss, J. Mol. Biol., 2004, 342(2), 571.

20. K. Palczewski, T. Kumasaka, T. Hori, C. A. Behnke, H. Motoshima, B. A. Fox, I. Le Trong, D. C. Teller, T. Okada, R. E. Stenkamp, M. Yamamoto and M. Miyano, Science, 2000, 289(5480), 739. 
21. V. Cherezov, J. Clogston, Y. Misquitta, W. Abdel-Gawad and M. Caffrey, Biophys. J., 2002, 83(6), 3393.

22. G. G. Prive and H. R. Kaback, J. Bioenerg. Biomemb., 1996, 28(1), 29.

23. M. A. Hanson and R. C. Stevens, Structure, 2009, 17(1), 8.

24. V. Cherezov, D. M. Rosenbaum, M. A. Hanson, S. G. F. Rasmussen, F. S. Thian, T. S. Kobilka, H. J. Choi, P. Kuhn, W. I. Weis, B. K. Kobilka and R. C. Stevens, Science, 2007, 318(5854), 1258.

25. V. P. Jaakola, M. T. Griffith, M. A. Hanson, V. Cherezov, E. Y. T. Chien, J. R. Lane, A. P. IJzerman and R. C. Stevens, Science, 2008, 322(5905), 1211.

26. T. Warne, M. J. Serrano-Vega, J. G. Baker, R. Moukhametzianov, P. C. Edwards, R. Henderson, A. G. W. Leslie, C. G. Tate and G. F. X. Schertler, Nature, 2008, 454(7203), 486.

27. J. H. Park, P. Scheerer, K. P. Hofmann, H. W. Choe and O. P. Ernst, Nature, 2008, 454(7201), 183.

28. P. Scheerer, J. H. Park, P. W. Hildebrand, Y. J. Kim, N. Krauß, H. W. Choe, K. P. Hofmann and O. P. Ernst, Nature, 2008, 455(7212), 497.

29. M. Murakami and T. Kouyama, Nature, 2008, 453(7193), 363.

30. V. R. Ratnala, Biotechnol. Lett., 2006, 28(11), 767.

31. D. Mustafi and K. Palczewski, Mol. Pharmacol., 2009, 75(1), 1.

32. S. Henikoff and J. G. Henikoff, Proc. Natl. Acad. Sci. U.S.A., 1992, 89(22), 10915.

33. M. A. Lomize, A. L. Lomize, I. D. Pogozheva and H. I. Mosberg, Bioinformatics, 2006, 22(5), 623.

34. S. J. Fleishman, V. M. Unger and N. Ben-Tal, Trends Biochem. Sci., 2006, 31(2), 106.

35. W. C. Wimley, T. P. Creamer and S. H. White, Biophys. J., 1996, 70(2), Tuam1.

36. T. Hessa, N. M. Meindl-Beinker, A. Bernsel, H. Kim, Y. Sato, M. Lerch-Bader, I. Nilsson, S. H. White and G. von Heijne, Nature, 2007, 450(7172), 1026.

37. M. B. Ulmschneider, M. S. P. Sansom and A. Di Nola, Proteins-Structure Function Bioinformatics, 2005, 59(2), 252.

38. S. H. White, Peptide Solvation H-Bonds, 2006, 72, 157.

39. F. Fanelli and P. G. De Benedetti, Chem. Rev., 2005, 105(9), 3297.

40. V. Yarov-Yarovoy, J. Schonbrun and D. Baker, Proteins-Structure Function Bioinformatics, 2006, 62(4), 1010.

41. N. Vaidehi, W. B. Floriano, R. Trabanino, S. E. Hall, P. Freddolino, E. J. Choi, G. Zamanakos and W. A. Goddard III, Proc. Natl. Acad. Sci. U.S.A., 2002, 99(20), 12622.

42. M. Y. S. Kalani, N. Vaidehi, S. E. Hall, R .J. Trabanino, P. L. Freddolino, M. A. Kalani, W. B. Floriano, V. W. T. Kam and W. A. Goddard III, Proc. Natl. Acad. Sci. U.S.A., 2004, 101(11), 3815.

43. P. L. Freddolino, M. Yashar, S. Kalani, N. Vaidehi, W. B. Floriano, S. E. Hall, R. J. Trabanino, V. W. T. Kam and W. A. Goddard III, Proc. Natl. Acad. Sci. U.S.A., 2004, 101(9), 2736. 
44. J. Y. Peng, N. Vaidehi, S. E. Hall and W. A. Goddard III, ChemMedChem, 2006, 1(8), 878.

45. N. Vaidehi, S. Schlyer, R. J. Trabanino, W. B. Floriano, R. Abrol, S. Sharma, M. Kochanny, S. Koovakat, L. Dunning, M. Liang, J. M. Fox, F. Lopes de Mendonça, J. E. Pease, W. A. Goddard III and R. Horuk, J. Biol. Chem., 2006, 281(37), 27613.

46. Y. Li, F. Zhu, N. Vaidehi, W. A. Goddard, III, F. Sheinerman, S. Reiling, I. Morize, L. Mu, K. Harris, A. Ardati and A. Laoui, J. Am. Chem. Soc., 2007, 129(35), 10720.

47. J. K. Bray and W. A. Goddard, 3rd, J. Mol. Graph. Model., 2008, 27(1), 66.

48. W. A. Goddard, III and R. Abrol, J. Nutr., 2007, 137(6 Suppl 1), 1528S, discussion 1548S.

49. W. A. Goddard, III, S. K. Kim, Y. Li, B. Trzaskowski, A. R. Griffith and R. Abrol, J. Structural Biol., 2010, 170(1), 10.

50. S. W. Englander and N. R. Kallenbach, Quarterly Rev. Biophys., 1983, 16(4), 521.

51. G. Swaminath, Y. Xiang, T. W. Lee, J. Steenhuis, C. Parnot and B. K. Kobilka, J. Biol. Chem., 2004, 279(1), 686.

52. A. M. D’Antona, K. H. Ahn and D. A. Kendall, Biochem., 2006, 45(17), 5606.

53. T. Kenakin, Annu. Rev. Pharmacol. Tox., 2002, 42, 349.

54. T. Kenakin and L. J. Miller, Pharmacol. Rev., 2010, 62(2), 265.

55. D. Spongier, C. Waeber, C. Pantaloni, F. Holsboer, J. Bockaert, P. H. Seeburgt and L. Journot, Nature, 1993, 365(6442), 170.

56. B. K. Kobilka and X. Deupi, Trends Pharmacol. Sci., 2007, 28(8), 397.

57. S. O. Smith, Ann. Rev. Biophys., 2010, 39, 309.

58. B. K. Kobilka, Biochim. Biophys. Acta-Biomembranes, 2007, 1768(4), 794.

59. X. J. Yao, C. Parnot, X. Deupi, V. R. P. Ratnala, G. Swaminath, D. Farrens and B. Kobilka, Nat. Chem. Biol., 2006, 2(8), 417.

60. M. P. Bokoch, Y. Zou, S. G. F. Rasmussen, C. W. Liu, R. Nygaard, D. M. Rosenbaum, J. J. Fung, H. J. Choi, F. S. Thian, T. S. Kobilka, J. D. Puglisi, W. I. Weis, L. Pardo, R. S. Prosser, L. Mueller and B. K. Kobilka, Nature, 2010, 463(7277), 108.

61. S. Bhattacharya, S. E. Hall and N. Vaidehi, J. Mol. Biol., 2008, 382(2), 539.

62. S. Bhattacharya, S. E. Hall, H. Li and N. Vaidehi, Biophys. J., 2008, 94(6), 2027.

63. R. Kondru, J. Zhang, C. Ji, T. Mirzadegan, D. Rotstein, S. Sankuratri and M. Dioszegi, Mol. Pharmacol., 2008, 73(3), 789.

64. K. Maeda, D. Das, H. Ogata-Aoki, H. Nakata, T. Miyakawa, Y. Tojo, R. Norman, Y. Takaoka, J. Ding, G. F. Arnold, E. Arnold and H. Mitsuya, J. Biol. Chem., 2006, 281(18), 12688.

65. T. Kenakin, Trends Pharmacol. Sci., 2007, 28(8), 407.

66. C. R. McCudden, M. D. Hains, R. J. Kimple, D. P. Siderovski and F. S. Willard, Cell. Mol. Life Sci., 2005, 62(5), 551.

67. Z. Selinger, Ann. Rev. Biochem., 2008, 77, xii.

68. L. Barki-Harrington and H. A. Rockman, Physiol., 2008, 23(1), 17. 
69. E. Hermans, Pharmacol. Ther., 2003, 99(1), 25.

70. L. M. Luttrell and R. J. Lefkowitz, J. Cell Sci., 2002, 115(3), 455.

71. S. J. Perry, G. S. Baillie, T. A. Kohout, I. McPhee, M. M. Magiera, K. L. Ang, W. E. Miller, A. J. McLean, M. Conti, M. D. Houslay and R. J. Lefkowitz, Science, 2002, 298(5594), 834.

72. C. D. Nelson, S. J. Perry, D. S. Regier, S. M. Prescott, M. K. Topham and R. J. Lefkowitz, Science, 2007, 315(5812), 663.

73. G. S. Baillie, A. Sood, I. McPhee, I. Gall, S. J. Perry, R. J. Lefkowitz and M. D. Houslay, Proc. Natl. Acad. Sci. U.S.A., 2003, 100(3), 940.

74. J. Hines, S. J. Fluharty and D. K. Yee, Biochem. Pharmacol., 2003, 66(2), 251.

75. K. Seta, M. Nanamori, J. G. Modrall, R. R. Neubig and J. Sadoshima, J. Biol. Chem., 2002, 277(11), 9268.

76. H. J. Wei, S. Ahn, S. K. Shenoy, S. S. Karnik, L. Hunyady, L. M. Luttrell and R. J. Lefkowitz, Proc. Natl. Acad. Sci. U.S.A., 2003, 100(19), 10782.

77. S. K. Shenoy, M. T. Drake, C. D. Nelson, D. A. Houtz, K. Xiao, S. Madabushi, E. Reiter, R. T. Premont, O. Lichtarge and R. J. Lefkowitz, J. Biol. Chem., 2006, 281(2), 1261.

78. D. Gesty-Palmer, M. Chen, E. Reiter, S. Ahn, C. D. Nelson, S. Wang, A. E. Eckhardt, C. L. Cowan, R. F. Spurney, L. M. Luttrell and R. J. Lefkowitz, J. Biol. Chem., 2006, 281(16), 10856.

79. S. Rajagopal, J. Kim, S. Ahn, S. Craig, C. M. Lam, N. P. Gerard, C. Gerard and R. J. Lefkowitz, Proc. Natl. Acad. Sci. U.S.A., 2010, 107(2), 628.

80. J. P. Pin, R. Neubig, M. Bouvier, L. Devi, M. Filizola, J. A. Javitch, M. J. Lohse, G. Milligan, K. Palczewski, M. Parmentier and M. Spedding, Pharmacol. Rev., 2007, 59(1), 5.

81. C. Romano, W. L. Yang and K. L. O’Malley, J. Biol. Chem., 1996, 271(45), 28612.

82. N. Kunishima, Y. Shimada, Y. Tsuji, T. Sato, M. Yamamoto, T. Kumasaka, S. Nakanishi, H. Jingami and K. Morikawa, Nature, 2000, 407(6807), 971.

83. D. Tsuchiya, N. Kunishima, N. Kamiya, H. Jingami and K. Morikawa, Proc. Natl. Acad. Sci. U.S.A., 2002, 99(5), 2660.

84. M. Tateyama, H. Abe, H. Nakata, O. Saito and Y. Kubo, Nat. Struct. Mol. Biol., 2004, 11(7), 637.

85. T. Galvez, B. Duthey, J. Kniazeff, J. Blahos, G. Rovelli, B. Bettler, L. Prézeau and J. P. Pin, Embo J., 2001, 20(9), 2152.

86. Y. Han, I. S. Moreira, E. Urizar, H. Weinstein and J. A. Javitch, Nat. Chem. Biol., 2009, 5(9), 688.

87. A. E. Brady and L. E. Limbird, Cell. Signalling, 2002, 14(4), 297.

88. S. L. Ferrari, D. D. Pierroz, V. Glatt, D. S. Goddard, E. N. Bianchi, F. T. Lin, D. Manen and M. L. Bouxsein, Endocrinol., 2005, 146(4), 1854.

89. R. W. Walters, A. K. Shukla, J. J. Kovacs, J. D. Violin, S. M. DeWire, C. M. Lam, J. R. Chen, M. J. Muehlbauer, E. J. Whalen and R. J. Lefkowitzet, J. Clin. Invest., 2009, 119(5), 1312.

90. G. C. Tsai and J. T. Coyle, Ann. Rev. Pharmacol. Tox., 2002, 42, 165.

91. T. W. Schwartz and B. Holst, Trends Pharmacol. Sci., 2007, 28(8), 366. 
92. J. M. Mathiesen, T. Ulven, L. Martini, L. O. Gerlach, A. Heinemann and E. Kostenis, Mol. Pharmacol., 2005, 68(2), 393.

93. K. J. Gregory, P. M. Sexton and A. Christopoulos, Curr. Neuropharmacol., 2007, 5(3), 157.

94. N. J. M. Birdsall and S. Lazareno, Mini-Rev. Med. Chem., 2005, 5(6), 523.

95. C. Valant, P. M. Sexton and A. Christopoulos, Mol. Intervent., 2009, 9(3), 125.

96. C. Valant, K. J. Gregory, N. E. Hall, P. J. Scammells, M. J. Lew, P. M. Sexton and A. Christopoulos, J. Biol. Chem., 2008, 283(43), 29312.

97. D. Schuster, C. Laggner and T. Langer, Curr. Pharmaceut. Design, 2005, 11(27), 3545 .

98. J. M. Bostwick, K. A. Hecksel, S. R. Stevens, J. H. Bower and J. E. Ahlskog, Mayo Clinic Proc., 2009, 84(4), 310.

99. M. J. Keiser, V. Setola, J. J. Irwin, C. Laggner, A. I. Abbas, S. J. Hufeisen, N. H. Jensen, M. B. Kuijer, R. C. Matos, T. B. Tran, R. Whaley, R. A. Glennon, J. Hert, K. L. H. Thomas, D. D. Edwards, B. K. Shoichet and B. L. Roth, Nature, 2009, 462(7270), 175. 